\title{
Funcionalización de los recubrimientos a base de quitosano para la conservación postcosecha de frutas y hortalizas
}

\author{
Luis M. Anaya-Esparza ${ }^{1,2}$, Alejandro Pérez-Larios ${ }^{2}$, \\ José M. Ruvalcaba-Gómez ${ }^{3}$, Jorge A. Sánchez-Burgos ${ }^{1}$, \\ Rafael Romero-Toledo ${ }^{4}$ y Efigenia Montalvo-González ${ }^{1 *}$ \\ ${ }^{1,2}$ Laboratorio Integral de Investigación en Alimentos, Tecnológico Nacional de México-Instituto \\ Tecnológico de Tepic. Av. Tecnológico \# 255 Fracc. Lagos del Country, Tepic 63175, Nayarit, México. \\ ${ }^{2}$ División de Ciencias Agropecuarias e Ingenierías, Centro Universitario de los Altos, Universidad de \\ Guadalajara, Tepatitlán de Morelos 47600, Jalisco, México. ${ }^{3}$ Campo Experimental Centro Altos de \\ Jalisco. Instituto Nacional de Investigaciones Forestales, Agrícolas y Pecuarias, Tepatitlán de Morelos \\ 47600, Jalisco, México. ${ }^{4}$ Universidad de Guanajuato, División de Ciencias Naturales y Exactas, \\ Guanajuato 36050, Guanajuato, México. E-mail: *emontalvo@ittepic.edu.mx
}

\begin{abstract}
RESUMEN
En años recientes, se ha buscado el desarrollo y aplicación de recubrimientos comestibles que sean seguros, biodegradables y con adecuadas propiedades tecnológicas y funcionales que ayuden a extender la vida de anaquel de frutas y hortalizas. El quitosano es uno de los biomateriales con mayor potencial para la elaboración de recubrimientos comestibles. Sin embargo, su principal desventaja es la alta permeabilidad al vapor de agua que exhibe, por lo que, una alternativa para mitigar esta limitante, es su funcionalización mediante la incorporación de compuestos orgánicos (aceites esenciales, extractos naturales, ácido ascórbico, hidrolizados de proteína, polisacáridos) e inorgánicos $\left(\mathrm{SiO}_{2}\right.$, $\mathrm{TiO}_{2}, \mathrm{ZnO}, \mathrm{Ag}$ y montmorillonita), además, de la adición de microorganismos (levaduras) a la matriz polimérica. El quitosano funcionalizado, aplicado a productos hortofrutícolas, ha mostrado mejores resultados (mayor vida de anaquel y cambios mínimos en parámetros de calidad) que los obtenidos al emplear quitosano sin funcionalizar. El objetivo de esta revisión es describir y discutir los beneficios y limitaciones de la funcionalización del quitosano y su aplicación en productos hortofrutícolas.

Palabras clave: recubrimientos comestibles, quitosano, funcionalización, protección, frutas y hortalizas, vida de anaquel.
\end{abstract}

\section{Functionalization of edible coating chitosan-based for fruits and vegetables postharvest preservation}

\begin{abstract}
In recent years, the development and application of safe and biodegradable edible coatings, with superior technological and functional properties have been studied to extend the shelf life of fresh fruit and vegetables. Chitosan is one of the most promising biomaterials for the development of edible coatings. However, the main disadvantage of this polysaccharide is related to the high water vapor permeability that it presents, therefore, an alternative to improve its performance, is its functionalization through the incorporation of organic (essential oils, natural extracts, ascorbic acid, protein hydrolysates, and polysaccharides) and inorganic compounds $\left(\mathrm{SiO}_{2}, \mathrm{TiO}_{2}, \mathrm{ZnO}, \mathrm{Ag}\right.$, and montmorillonite), but also, the addition of microorganisms (yeast) in the chitosan matrix. The application of edible functionalized-chitosan coatings on fruits and vegetables has given better results (significant prolongation of the shelf life and minimal changes in quality parameters) than those obtained when pure chitosan-coating was applied. This review describes the advantages and limitations of functionalization of edible chitosan films in the preservation of post-harvest of fruit and vegetables. Keywords: edible coatings, chitosan, functionalization, protection, fruits and vegetables, shelf life.
\end{abstract}




\section{INTRODUCCIÓN}

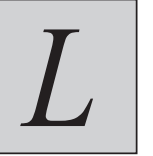

as frutas y hortalizas son tejidos vivos que están expuestos a un deterioro progresivo después de ser cosechados, ocasionado tanto por factores intrínsecos (fisiológicos y bioquímicos) como por factores extrínsecos (manejo, transporte y almacenamiento), además de la interacción con microorganismos patógenos causantes de distintas enfermedades en los mismos (Departamento de Agricultura de los Estados Unidos, USDA por sus siglas en inglés) (USDA, 2016). En este sentido, se estima que las pérdidas postcosecha a nivel mundial de frutas y hortalizas se sitúan entre el 30 y $50 \%$ de la producción total (Porat, Lichter, Terry, Harker \& Buzby, 2018), por lo que, su conservación representa un reto a nivel mundial (USDA, 2016; Rodríguez-Guzmán, González-Estrada, BautistaBaños \& Gutiérrez-Martínez, 2019). Con esta finalidad se han desarrollado y aplicado diversas tecnologías (bajas temperaturas, empaques con atmósferas modificadas, envasado al vacío y aditivos químicos), entre ellas la aplicación de recubrimientos comestibles, que actúan como una barrera física que protege al fruto de los factores ambientales, prolonga su vida de anaquel y le confiere valor agregado a los productos hortofrutícolas (USDA, 2016).

Los recubrimientos comestibles pueden ser elaborados a partir de proteínas, lípidos y/o polisacáridos. Los polisacáridos como el almidón, celulosa, pectina, alginato y quitosano destacan como los materiales más empleados para su elaboración (Rinaudo, 2014; Solano-Doblado, Alamilla-Beltrán \& JiménezMartínez, 2018). Debido a sus propiedades antimicrobianas y de inducción de mecanismos de defensa en frutos, así como a sus propiedades tecnológicas (solubilidad, compatibilidad con compuestos orgánicos e inorgánicos y habilidad para formación de recubrimientos), el quitosano es una de las alternativas viables para la elaboración de recubrimientos comestibles (BerumenVarela, Coronado-Partida, Ochoa-Jiménez, Chacón-López \& Gutiérrez-Martínez, 2015; Bautista-Baños, Ventura-Aguilar, Correa-Pacheco \& Corona-Rangel, 2017).

Investigaciones recientes se han centrado en la funcionalización del quitosano para la conservación de frutas y hortalizas, con lo que se ha demostrado que la incorporación de diversos compuestos orgánicos, inorgánicos y/o biológicos dentro de la matriz del quitosano ha mejorado y/o potencializado las propiedades tecnológicas y funcionales de este tipo de recubrimientos (permeabilidad al vapor de agua e intercambio de gases, propiedades mecánicas, actividad antimicrobiana y antioxidante), con lo que se han obtenido mejores resultados en términos de conservar la calidad de los frutos en los que se han utilizado, en comparación con la aplicación individual del quitosano o en frutos sin recubrimiento (Gol, Patel \& RamanaRao, 2013; Fortunati et al., 2017; Hajji, Younes, Affes, Boufi \& Nasri, 2018). El objetivo de esta revisión fue recopilar, describir y discutir los beneficios y limitaciones de la funcionalización con compuestos orgánicos, inorgánicos y/o biológicos de los recubrimientos comestibles a base de quitosano y su aplicación en productos hortofrutícolas.

\section{TeCnOlOgías de CONSERVACIÓN E ÍNDICES DE CALIDAD EN FRUTOS POSTCOSECHA}

En la actualidad, se han estudiado diversas tecnologías para prolongar la vida útil de los productos hortofrutícolas como el uso de bajas temperaturas (refrigeración y congelación), inmersión en aditivos químicos, atmósferas modificadas, envasado al vacío y recubrimientos comestibles (de manera individual o en combinación). La finalidad de estas tecnologías es minimizar las pérdidas ocasionadas durante el manejo, transporte y almacenamiento postcosecha de los productos hortofrutícolas (USDA, 2016).

Actualmente, existen diversos índices utilizados para determinar la calidad de los productos hortofrutícolas y cambios postcosecha, los más importantes tienen relación con los parámetros fisiológicos (tasa de respiración y la producción de etileno), microbiológicos (presencia de microorganismos patógenos), físicos (pérdida de peso y firmeza) y fisicoquímicos ( $\mathrm{pH}$, acidez titulable y sólidos solubles totales) de los frutos (USDA, 2016). Asimismo, debe considerarse que durante y después de la cosecha, se pueden presentar cambios fisiológicos importantes en los frutos, algunos de estos son dependientes de la presencia de oxígeno, por ejemplo, la presencia de microorganismos fitopatógenos en la superficie del fruto puede promover un aumento en la tasa de respiración y la producción de etileno, lo que conduce a una mayor emisión de calor, incremento en la tasa metabólica y de descomposición en el fruto(USDA, 2016). Por lo que, una alternativa para minimizar estas reacciones metabólicas es la aplicación de recubrimientos comestibles (Bautista-Baños et al., 2017; Solano-Doblado et al., 2018). Adicionalmente, debe considerarse que cada tecnología tiene sus propias ventajas y limitaciones, ya que la eficacia de los tratamientos de conservación depende directamente de la interacción frutocontaminación-almacenamiento (USDA, 2016).

\section{ASPECTOS GENERALES DE LOS RECUBRIMIENTOS COMESTIBLES}

Los recubrimientos comestibles son matrices complejas que, aplicadas al fruto, funcionan como una barrera protectora frente a factores ambientales (contaminación y microorganismos patógenos), y a su vez como una membrana semipermeable que permite el intercambio de gases (reducción de la disponibilidad de $\mathrm{O}_{2}$ e incremento de $\mathrm{CO}_{2}$ ), lo que favorece la creación de una atmósfera modificada entre el fruto y el recubrimiento que, principalmente, desacelera el metabolismo natural de los productos hortofrutícolas (tasa de respiración, producción de etileno), lo que resulta en una menor deshidratación y pérdida fisiológica de peso de los mismos (USDA, 2016; Solano-Doblado et al., 2018; Hajji et al., 2018; Jiao et al., 2019). 
Un recubrimiento comestible es una capa delgada formada sobre el alimento, que puede ser aplicada por inmersión o aspersión (Solano-Doblado et al., 2018; Jiao et al., 2019). Estos pueden ser elaborados a partir de proteínas, lípidos y/o polisacáridos (solos o en combinación) provenientes de diferentes fuentes naturales (exoesqueleto de crustáceos, proteínas de soya y leche, almidón, pectinas, mucílago de nopal y gomas naturales) (Rinaudo, 2014; Solano-Doblado et al., 2018). La elección del material con el que se elaboran los recubrimientos estará en función del desempeño y características intrínsecas de cada material, como lo son costos, disponibilidad, propiedades mecánicas (tensión y flexibilidad) y ópticas (brillo, opacidad, transparencia), estabilidad, solubilidad, permeabilidad al vapor de agua y capacidad de intercambio de gases, adherencia al fruto y protección contra microorganismos patógenos o los asociados al deterioro del fruto. Dichos recubrimientos, deberán ser inocuos, biodegradables, incoloros e insípidos (USDA, 2016). Con base en esos criterios, uno de los materiales preferidos para este fin son los polisacáridos, de entre los cuales destaca el quitosano (Solano-Doblado et al., 2018; Mujtaba et al., 2019; MaderaSantana et al., 2019).

\section{QUITOSANO COMO RECUBRIMIENTO COMESTIBLE}

El quitosano es un heteropolisacárido formado por unidades de N-acetil-D-glucosamina mediante enlaces glicosídicos ( $\beta$ 1-4), que destaca por su biodegradabilidad, biocompatibilidad, funcionalidad, nula toxicidad, alta adherencia, propiedades antimicrobianas y capacidad para formar recubrimientos (Rinaudo, 2008; Bautista-Baños et al., 2017; Jiao et al., 2019; Mujtaba et al., 2019). Es un biopolímero de naturaleza policatiónica debido a la presencia de grupos amino en su estructura, los cuales juegan un papel importante dentro de las propiedades fisicoquímicas y biológicas del quitosano (Rinaudo, 2008; Berumen-Varela et al., 2015; BautistaBaños et al., 2017). Se ha propuesto que las interacciones electrostáticas entre el grupo amino (que presenta alta densidad de carga) del quitosano con los grupos fosforilo presentes en los lípidos de membrana de los microorganismos son los principales responsables de la actividad antimicrobiana de este biopolímero (Bautista-Baños et al., 2017). Además, este grupo funcional, junto con los grupos hidroxilo $(-\mathrm{OH})$ presentes en la estructura del quitosano pueden actuar como sitios activos para la interacción y/o adsorción de compuestos orgánicos e inorgánicos (Mohandas, Deepthi, Biswas \& Jayakumar, 2017).

El quitosano puede obtenerse a partir de fuentes naturales (exoesqueleto de crustáceos, paredes celulares de hongos $\mathrm{y}$ algas) por medios químicos $\mathrm{y} / \mathrm{o}$ enzimáticos a través de la desacetilación de la quitina (Bautista-Baños et al., 2017; Mujtaba et al., 2019). De acuerdo con la eficiencia y naturaleza del método de obtención, este biopolímero puede presentar diferentes grados de desacetilación (GDA) y peso molecular, lo anterior asociado al porcentaje de oxidación del quitosano y a la severidad de los procesos de desproteinización y desmineralización de cada método de obtención, además puede existir una despolimerización parcial de la quitina y por consiguiente resultar en un quitosano con peso molecular bajo (López, Rivas, Loaiza \& Sabino, 2010). El GDA del quitosano determina la cantidad de grupos aminos libres que posee (a mayor GDA, mayor cantidad de grupos amino libres), lo que influye en las propiedades tecnológicas y funcionales del recubrimiento (Chen, Wang, Lai \& Lin, 2003; Rinaudo, 2008; Mujtaba et al., 2019). Por ejemplo, la viscosidad de las soluciones del quitosano es influenciada por el GDA del biopolímero, como lo reportan Chen et al. (2003), que al utilizar quitosano con un GDA alto (> $85 \%$ ), la viscosidad de las soluciones formadoras de recubrimientos es baja $(<5$ unidades de viscosidad rápida, UVR) en comparación con la obtenida al emplear quitosano con bajo $(50 \%=30$ UVR) y medio ( $75 \%=15$ UVR) GDA, lo anterior, atribuido al incremento en la formación de puentes de hidrogeno con el medio, promovido por el incremento en las cargas positivas de los grupos amino del quitosano. En este contexto, se ha recomendado que para elaborar recubrimientos de quitosano uniformes y estables se empleen soluciones con baja viscosidad, debido a que una solución con una viscosidad alta es difícil de manejar y genera recubrimientos poco uniformes, con presencia de burbujas de aire, lo que afecta la calidad del mismo (Wu et al., 2016).

Los recubrimientos a base de quitosano se pueden preparar a partir de la disolución del quitosano en medios acidificados (preferentementeácidos orgánicos) (Bautista-Baños etal.,2017) y se han empleado en la conservación de frutas y hortalizas debido a su actividad antifúngica (Berumen-Varela et al., 2015; Fortunati et al., 2017). Asimismo, el quitosano puede actuar como agente inductor de diferentes mecanismos de defensa del fruto (Romanazzi et al., 2016; Gutiérrez-Martínez, BautistaBaños, Berumen-Varela, Ramos-Guerrero \& Hernández-Ibáñez, 2017). Berumen-Varela et al. (2015) mencionan que el quitosano aplicado en frutos de mango, tiene un efecto positivo sobre la expresión y actividad de la enzima peroxidasa, que reduce los daños asociados a la presencia de microorganismos patógenos. Además, el análisis del transcriptoma de la interacción aguacateColletotrichum-quitosano, demostró que el quitosano induce la expresión de genes relacionados en la biosíntesis de compuestos fenólicos y regula un gran número de procesos metabólicos, que previenen la proliferación del fitopatógeno (Xoca-Orozco et al., 2017). Por su parte, Zhang, Wang, Hu and Liu (2015a) reportaron que la aplicación de quitosano al $0.2 \%$ en jitomate cherry induce la expresión de los genes MPK3, MPK6, PR1a1 y PR5, que le confiere al fruto resistencia en contra del moho gris causado por Botrytis cinerea, posiblemente asociado con la vía de señalización de la proteína quinasa activada por mitógeno (MAPK).

Factores como la viscosidad de la solución, temperatura de secado, modo de preparación, la concentración y tipo de ácido utilizado para su disolución, así como la concentración y el grado 
de desacetilación del quitosano, pueden limitar la efectividad de los recubrimientos resultantes, principalmente en términos de actividad antimicrobiana, rigidez y permeabilidad de gases (Rinaudo, 2008; Bautista-Baños et al., 2017; Jiao et al., 2019). Una alternativa para mejorar el desempeño (propiedades tecnológicas y funcionales) del quitosano como recubrimiento, es su funcionalización mediante la incorporación de diversos componentes orgánicos, inorgánicos y/o biológicos dentro de la matriz polimérica (Hajji et al., 2018; Kaewklin, Siripatrawan, Suwanagul \& Lee, 2018; Jiao et al., 2019; Wang, et al., 2019).

\section{FUnCIONALIZACIÓN DEL QUITOSANO PARA LA CONSERVACIÓN DE FRUTAS Y HORTALIZAS}

La funcionalización se define como la modificación de las propiedades fisicoquímicas de cualquier material mediante la incorporación de otro (Bilalis, Katsigiannopoulos, Avgeropoulos \& Sakellariou, 2014). En general, existen dos tipos de funcionalización, covalente y no covalente (Layek \& Nandi, 2013). La funcionalización covalente, se basa en la unión mediante enlaces covalentes y por reacción directa de los grupos funcionales de los diversos componentes, lo que en la mayoría de los casos, modifica las propiedades intrínsecas del material funcionalizado, mientras que la funcionalización no covalente o supramolecular, se basa en la adsorción física del material funcionalizante en la red (puede ser en la superficie) del material funcionalizado mediante interacciones electrostáticas o hidrofóbicas, sin alterar las características intrínsecas del material funcionalizado (Bilalis et al., 2014; Maccora et al., 2019).

En la actualidad existe un creciente interés por el desarrollo de tecnologías y/o materiales que permitan incrementar la vida de anaquel de los productos hortofrutícolas (USDA, 2016). Por lo que, la funcionalización del quitosano mediante la adición de compuestos orgánicos (compuestos bioactivos, ácidos orgánicos, aceites esenciales), inorgánicos $\left(\mathrm{TiO}_{2}, \mathrm{SiO}_{2}, \mathrm{Ag}\right)$, y microorganismos (levaduras y lactobacilos), además de la integración de otras biomoléculas como proteínas, polisacáridos y lípidos a la matriz polimérica mediante interacciones covalentes o no covalentes (Figura 1) puede ser una alternativa viable para mejorar las propiedades tecnológicas, funcionales y biológicas de este biopolímero (Rinaudo, 2008; Bautista-Baños et al., 2017; Solano-Doblado et al., 2018).

En la Tabla I se presentan algunos de los hallazgos más recientes relacionados con el uso de quitosano funcionalizado (RQF) con diferentes compuestos orgánicos e inorgánicos, así como su aplicación y principales efectos sobre los parámetros de calidad (fisicoquímicos, fisiológicos y microbiológicos), atributos sensoriales y vida de anaquel de frutas y hortalizas.

\section{FUNCIONALIZACIÓN CON ACEITES ESENCIALES}

La incorporación de aceites esenciales de canela, naranja y limón a recubrimientos a base de quitosano ha demostrado ser efectiva para prolongar la vida de anaquel de fresas (7-15 días a $\left.5{ }^{\circ} \mathrm{C}\right)$, pepinos $\left(21\right.$ días a $\left.10^{\circ} \mathrm{C}\right)$, jujube $(20$ días a 4 ${ }^{\circ} \mathrm{C}$ ), pimiento morrón $\left(35\right.$ días a $8{ }^{\circ} \mathrm{C}$ ) y mango (11 días a $4{ }^{\circ} \mathrm{C}$ ) (Xing et al., 2011a; Xing, Xu, Che, Li \& Li, 2011b; Perdones, Sánchez-González \& Vargas, 2012; Rico, Gutiérrez \& Díaz-Moreno, 2012; López-Mata et al., 2012; Mohammadi, Hashemi \& Hosseini, 2015). En general, los frutos tratados con los recubrimientos funcionalizados con aceites esenciales disminuyen su tasa de respiración, mantienen su firmeza y calidad $(\mathrm{pH}$, sólidos solubles totales, acidez titulable y color). Además, reducen la incidencia y aparición de signos de enfermedad causada por los hongos $B$. cinerea en fresa (Perdones et al., 2012) y Penicillium citrinum en frutos de jujube (Xing et al., 2011b) y por el oomiceto Phytophthora drechsleri en pepino (Mohammadi et al., 2015), y les provee protección contra la proliferación de bacterias (reducción de coliformes, bacterias mesófilas aerobias y bacterias psicrófilas) durante su almacenamiento, en comparación con los frutos

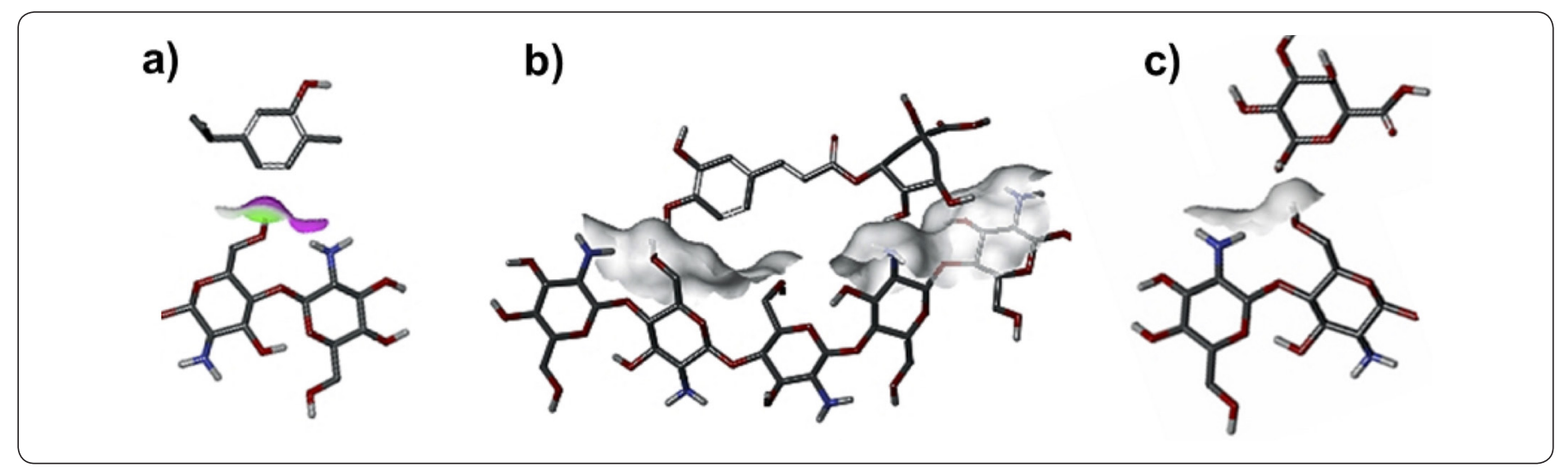

Figura 1. Posible interacción estructural del quitosano funcionalizado con a) carvacrol, b) ácido clorogénico y c) pectina.

Fuente: Elaboración propia. Estructuras tomadas de la base de datos PubChem, National Center for Biotechnology Information (quitosano ID: 71853, carvacrol ID: 10364, ácido clorogénico ID: 1794427, pectina: 441476) y modificadas con uso del software Discovery Studio Predictive Science Application (Dassault Systèmes BIOVIA®). 
https://doi.org/10.22201/fesz.23958723e.2020.0.241

\begin{tabular}{|c|c|c|c|c|c|c|c|c|c|c|c|c|c|}
\hline 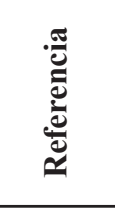 & 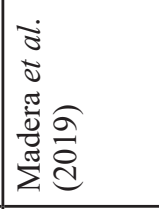 & 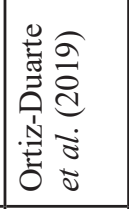 & 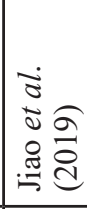 & 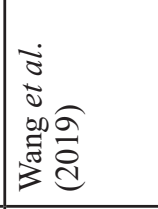 & 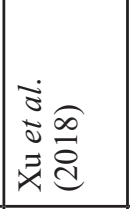 & 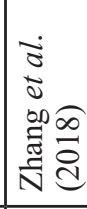 & 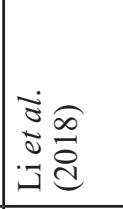 & 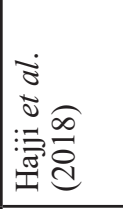 & 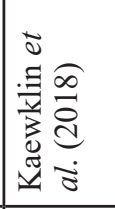 & 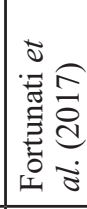 & 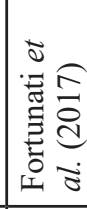 & 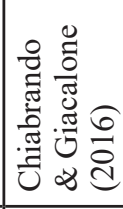 & 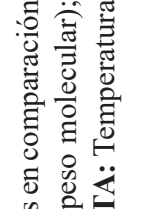 \\
\hline 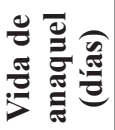 & 인 & 9 & $\infty$ & $\stackrel{\infty}{\sim}$ & $=$ & F & $\approx$ & $=$ & $\because$ & r & 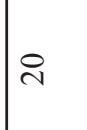 & a & 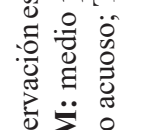 \\
\hline 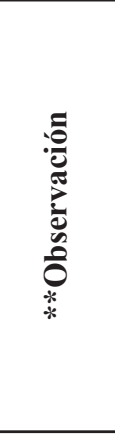 & 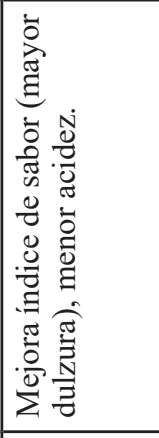 & 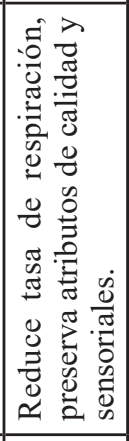 & 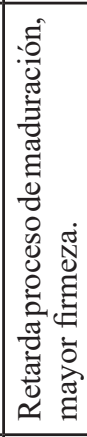 & 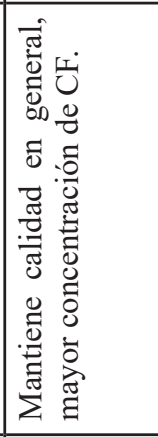 & 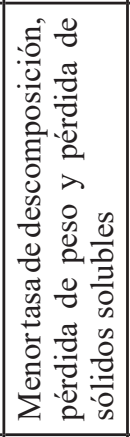 & 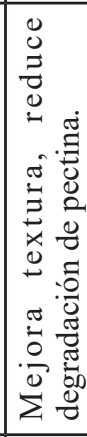 & 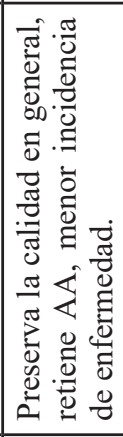 & 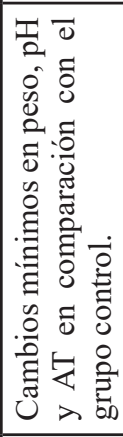 & 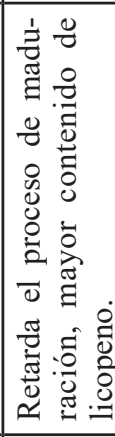 & 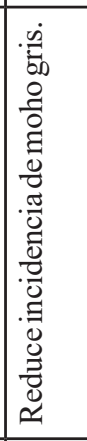 & 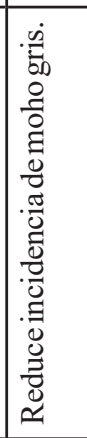 & 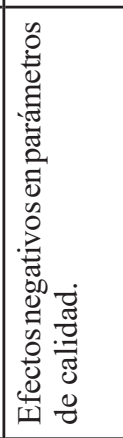 & 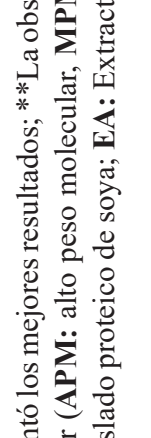 \\
\hline 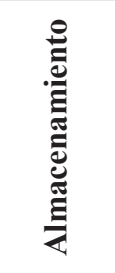 & $\begin{array}{l}0 \\
0 \\
+\end{array}$ & 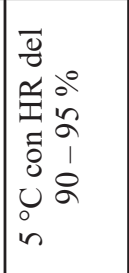 & 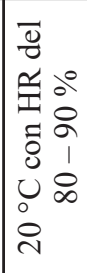 & 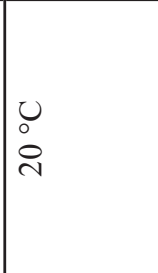 & 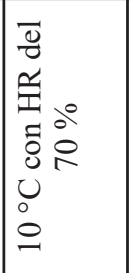 & 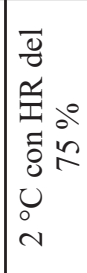 & 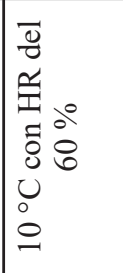 & $\begin{array}{l}0 \\
0 \\
i 2\end{array}$ & 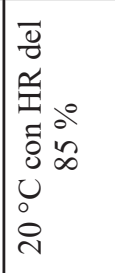 & 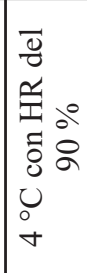 & 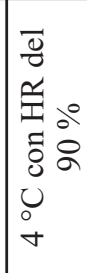 & $\begin{array}{l}0 \\
0 \\
+\end{array}$ & 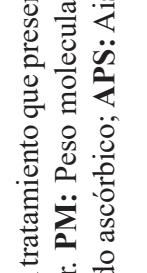 \\
\hline 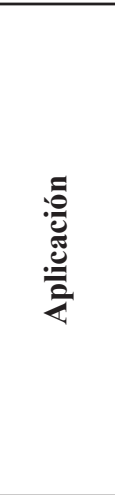 & 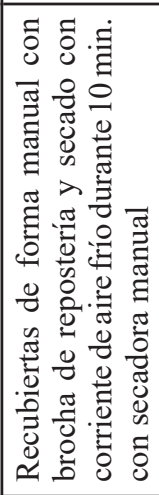 & 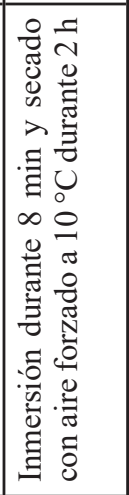 & 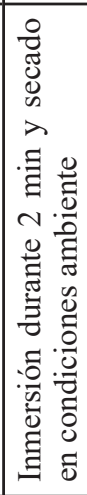 & 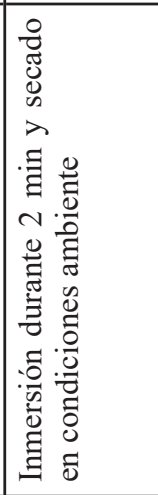 & 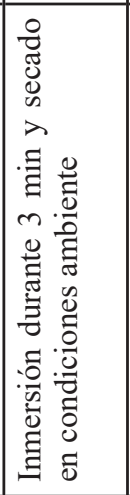 & 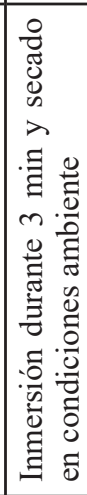 & 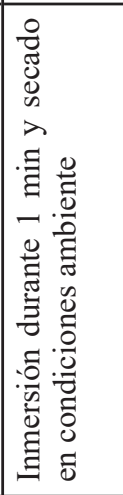 & 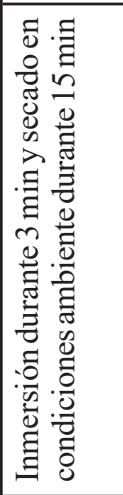 & 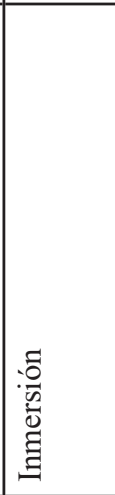 & 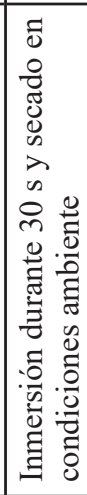 & 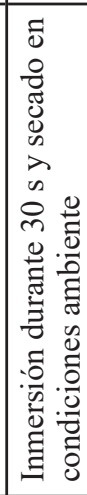 & 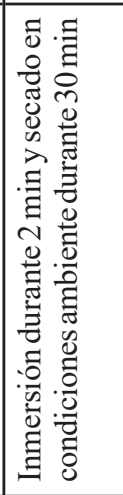 & \\
\hline 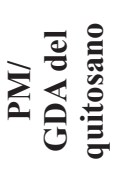 & 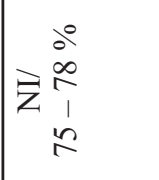 & $\begin{array}{l}\partial^{0} \\
i n \\
\text { vi } \\
i z \\
z\end{array}$ & 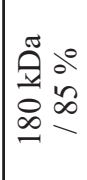 & $\mid \begin{array}{l}0 \\
0 \\
\infty \\
\dot{z}\end{array}$ & 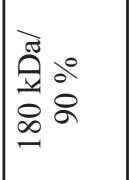 & $\begin{array}{l}\partial^{\circ} \\
8 \\
2 \\
\wedge 1 \\
\hat{Z}\end{array}$ & $\begin{array}{l}\therefore 0 \\
2 \\
2 \\
11 \\
\hat{z}\end{array}$ & 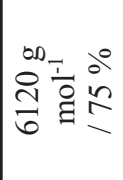 & 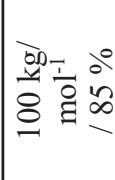 & 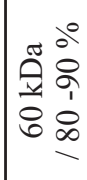 & 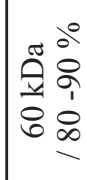 & $\mid \begin{array}{l}\bar{z} \\
\bar{z}\end{array}$ & \\
\hline 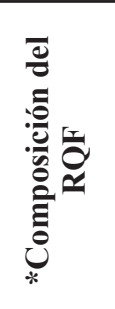 & 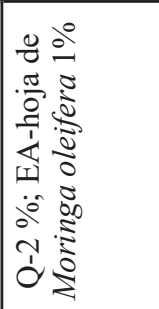 & 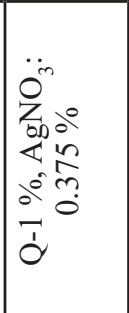 & 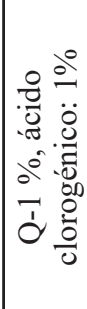 & 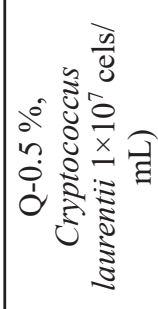 & 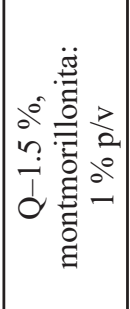 & 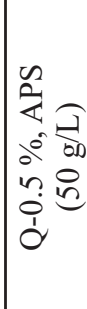 & 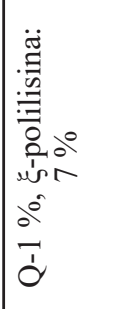 & 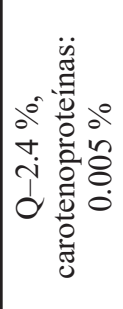 & 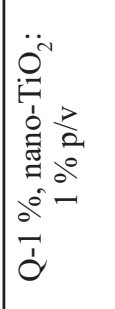 & 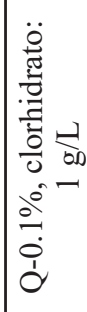 & 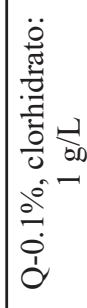 & 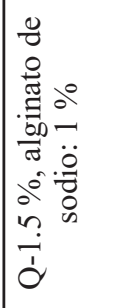 & \\
\hline 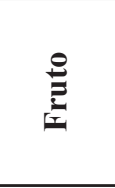 & 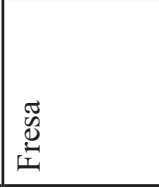 & $\frac{.00}{\sum^{\frac{0}{2}}}$ & 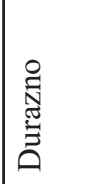 & $\mid \begin{array}{l}. \frac{\pi}{3} \\
0 \\
0 \\
0 \\
0\end{array}$ & 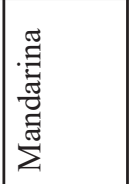 & 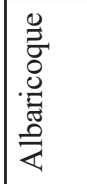 & 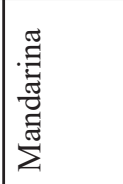 & $\tilde{g}$ & 泀 & $\stackrel{00}{\Xi}$ & 文 & 言 & \\
\hline
\end{tabular}




\begin{tabular}{|c|c|c|c|c|c|c|c|c|c|c|c|c|c|}
\hline 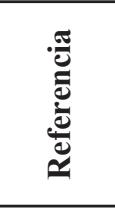 & 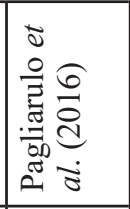 & 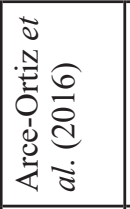 & 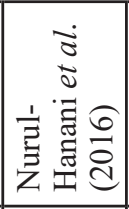 & 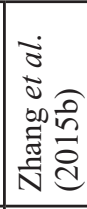 & 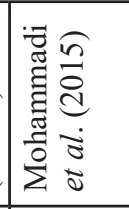 & 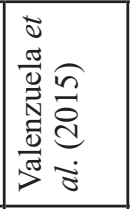 & 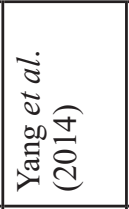 & 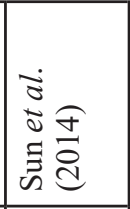 & 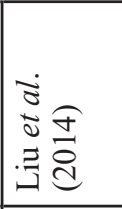 & 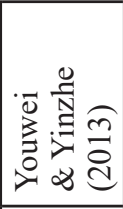 & 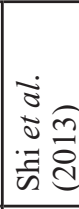 & 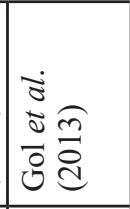 & 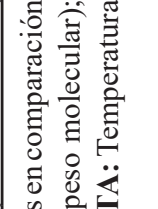 \\
\hline 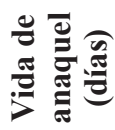 & 10 & \pm & $\vec{m}$ & $\simeq$ & $\vec{\sim}$ & $\stackrel{2}{2}$ & $\ddot{n}$ & $r$ & ㄱ. & $\stackrel{2}{2}$ & $\infty$ & $\approx$ & है \\
\hline 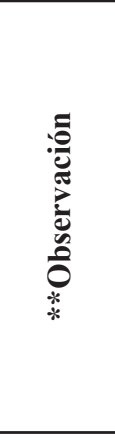 & 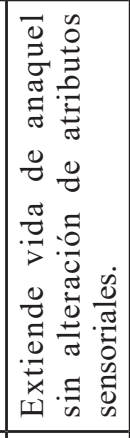 & 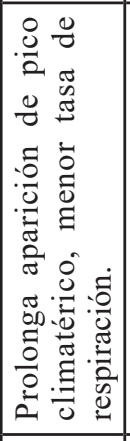 & 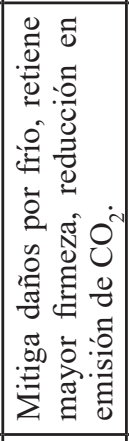 & 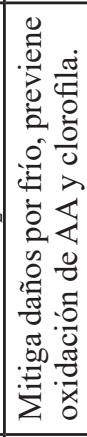 & 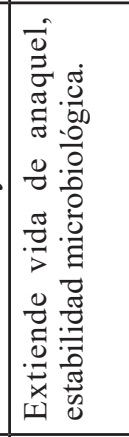 & 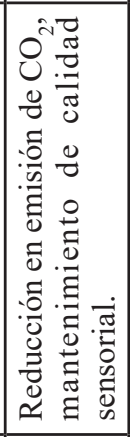 & 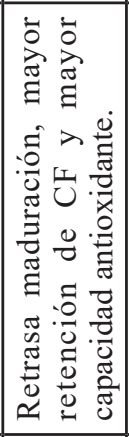 & 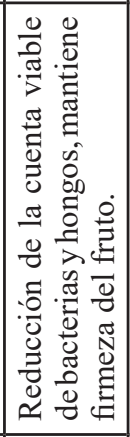 & 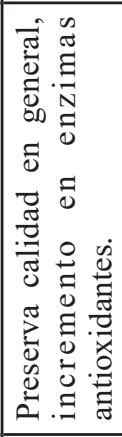 & 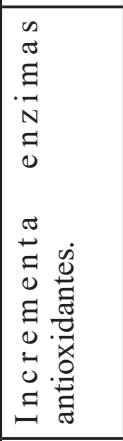 & 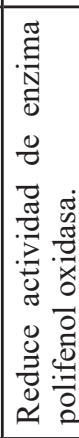 & 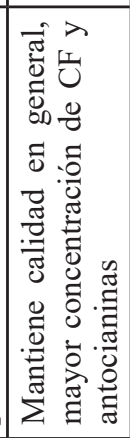 & 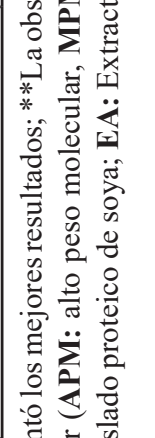 \\
\hline 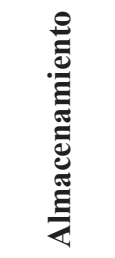 & $\begin{array}{l}0 \\
0 \\
+\end{array}$ & 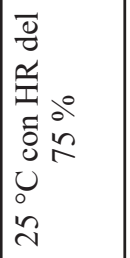 & 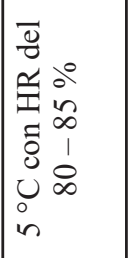 & 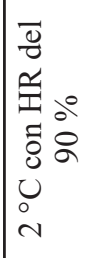 & 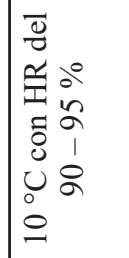 & 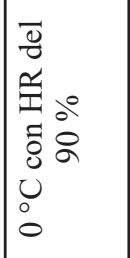 & 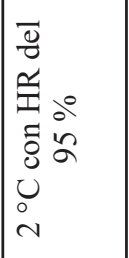 & $\begin{array}{l}0 \\
0 \\
0\end{array}$ & 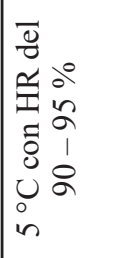 & 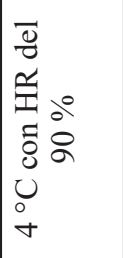 & 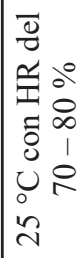 & 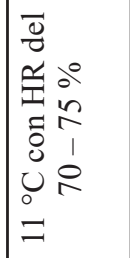 & 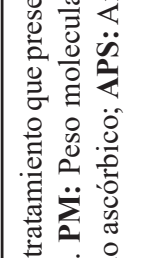 \\
\hline & 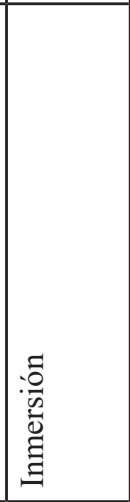 & 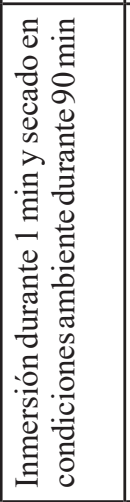 & 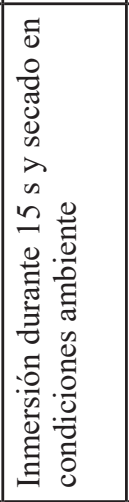 & 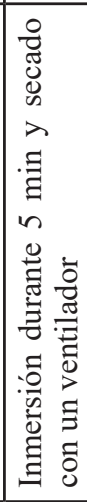 & 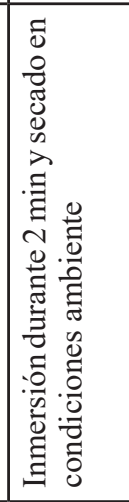 & 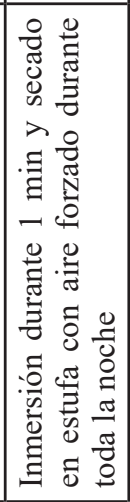 & 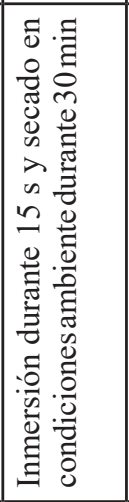 & 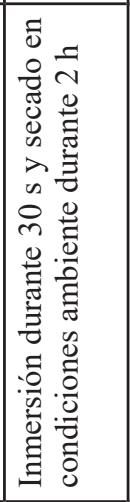 & 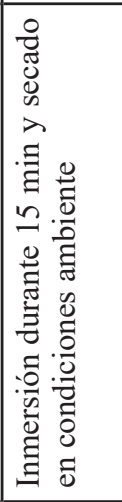 & 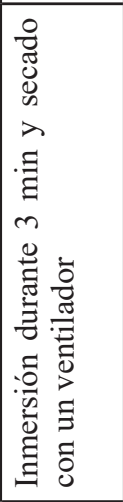 & 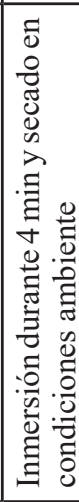 & 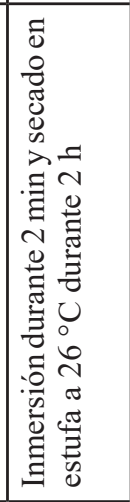 & \\
\hline 离 & 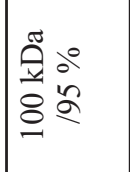 & $\begin{array}{l}\dot{0} \\
\vdots \\
2 \\
z\end{array}$ & $\sum_{i}^{z}$ & $\begin{array}{l}\therefore 0 \\
\dot{2} \\
2 \\
11 \\
z\end{array}$ & 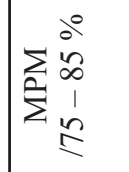 & 妾 & $\begin{array}{l}20 \\
8 \\
\alpha 1 \\
11 \\
z\end{array}$ & 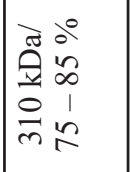 & $\mid \begin{array}{l}\bar{z} \\
\bar{z}\end{array}$ & $\begin{array}{l}0^{0} \\
i \\
\infty \\
z\end{array}$ & 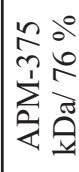 & $\sum_{\substack{\alpha \\
\dot{\alpha}}}^{a^{0}}$ & \\
\hline 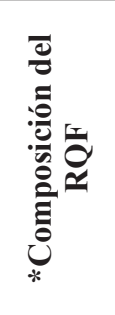 & 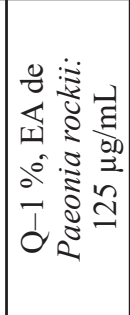 & 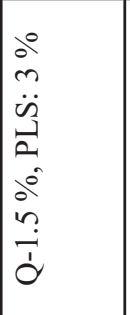 & 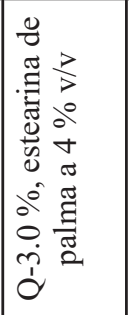 & 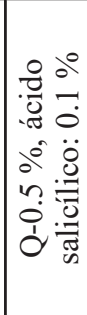 & 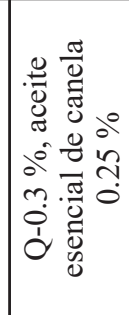 & 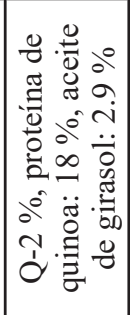 & 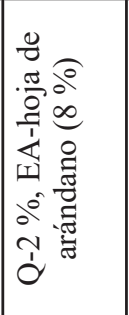 & 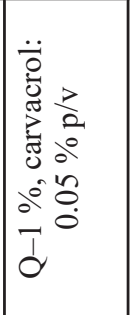 & 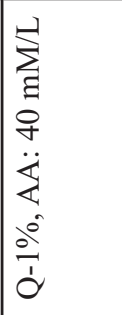 & 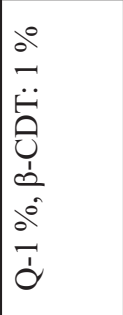 & 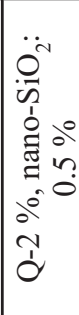 & $\begin{array}{l}0 \\
0 \\
0 \\
\sum_{0} \\
0 \\
0 \\
0 \\
0 \\
0\end{array}$ & \\
\hline 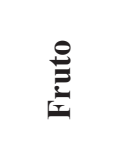 & $\widetilde{W}$ & 迨 & 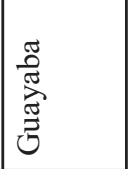 & 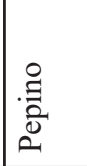 & : & 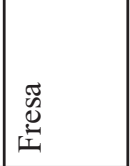 & 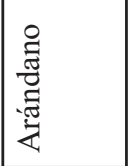 & 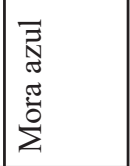 & 童 & 家 & 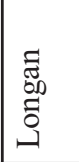 & 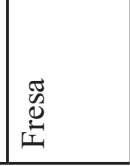 & \\
\hline
\end{tabular}


https://doi.org/10.22201/fesz.23958723e.2020.0.241

\begin{tabular}{|c|c|c|c|c|c|c|c|c|c|c|c|c|c|}
\hline 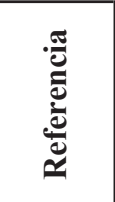 & 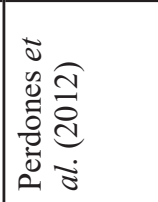 & 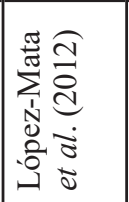 & 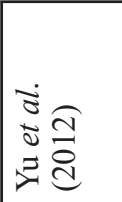 & 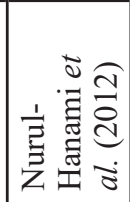 & 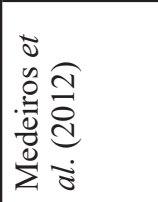 & 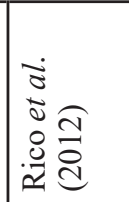 & 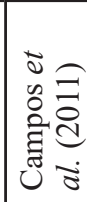 & 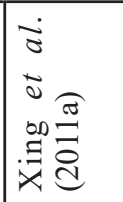 & 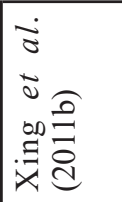 & 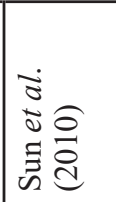 & 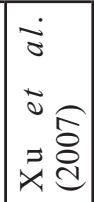 & 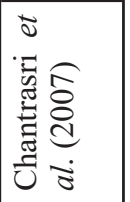 & 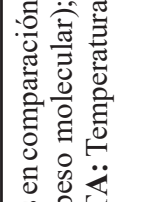 \\
\hline 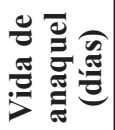 & $r$ & \pm & กี & ㄱ. & f & $\approx$ & $a$ & m & શે & ஜ & $r$ & $r$ & \\
\hline 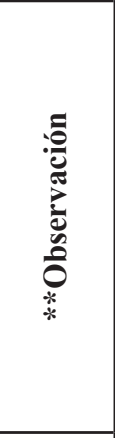 & 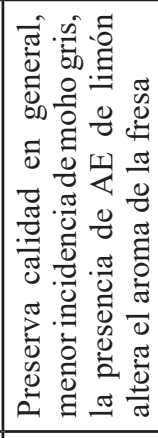 & 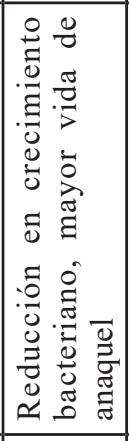 & 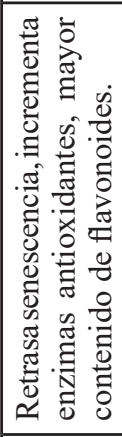 & 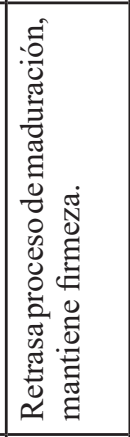 & 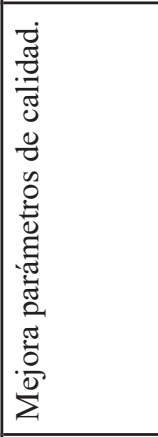 & 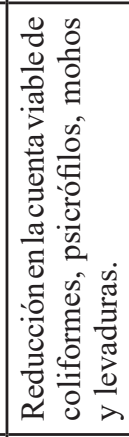 & 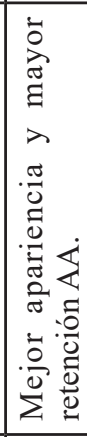 & 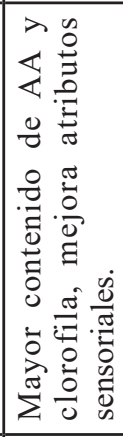 & 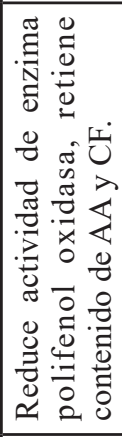 & 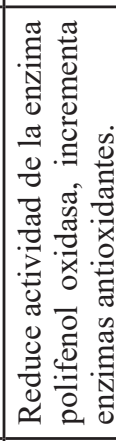 & 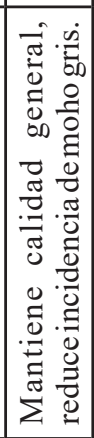 & 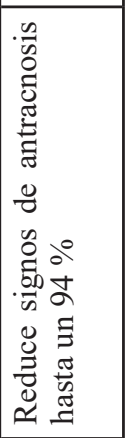 & $\exists$ \\
\hline 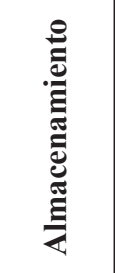 & 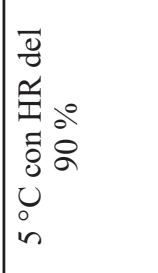 & $\begin{array}{l}0 \\
\text { in }\end{array}$ & 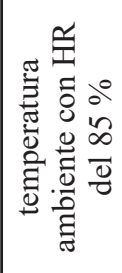 & $\begin{array}{l}0 \\
0 \\
\infty \\
\infty\end{array}$ & 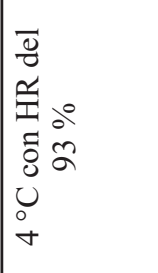 & $\begin{array}{l}0 \\
0 \\
+\end{array}$ & $\begin{array}{l}0 \\
0 \\
0\end{array}$ & 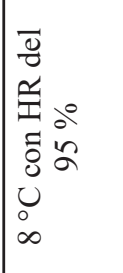 & 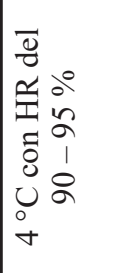 & 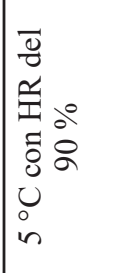 & $\begin{array}{l}0 \\
0 \\
n\end{array}$ & 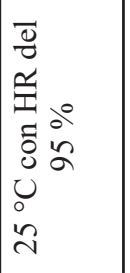 & \\
\hline 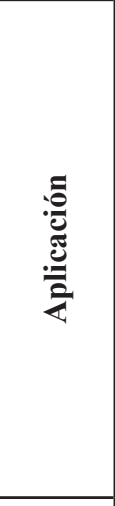 & 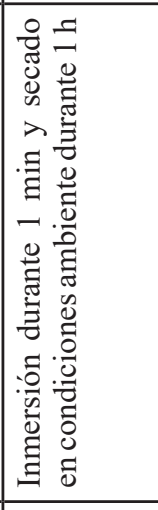 & 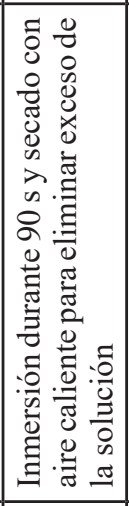 & 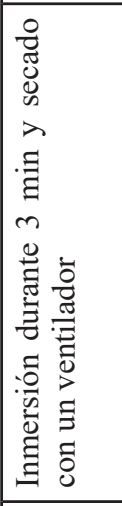 & 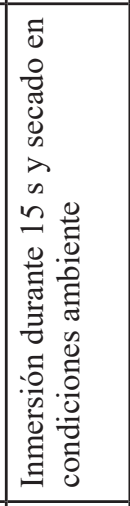 & 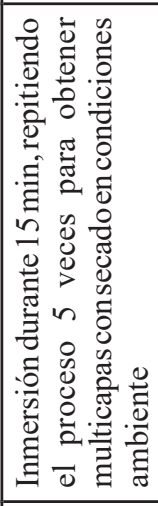 & 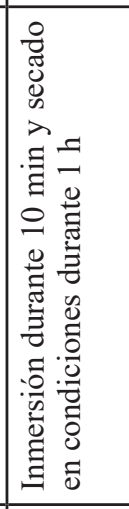 & 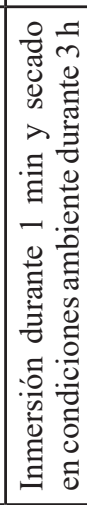 & 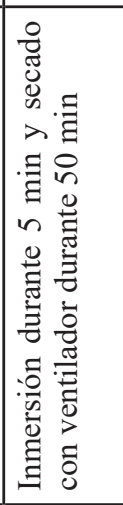 & 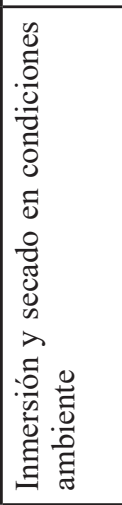 & 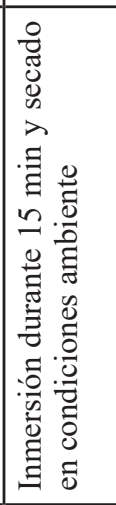 & 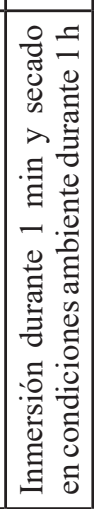 & 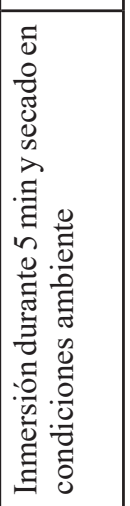 & \\
\hline 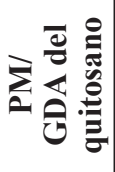 & $\begin{array}{l}\sum_{i} i_{0}^{0} \\
\dot{2}\end{array}$ & 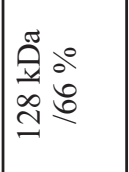 & 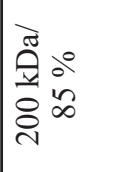 & $\begin{array}{l}00 \\
i n \\
\infty \\
z\end{array}$ & $\begin{array}{l}z \\
z \\
z\end{array}$ & Z & $\begin{array}{l}\partial^{0} \\
\infty \\
2 \\
z\end{array}$ & $\begin{array}{l}20 \\
2 \\
\alpha \\
11 \\
\hat{z}\end{array}$ & $\begin{array}{l}20 \\
i \\
\alpha \\
11 \\
z\end{array}$ & 文 & 盲 & 文 & \\
\hline 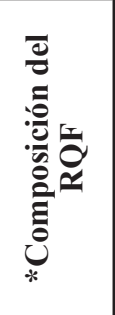 & 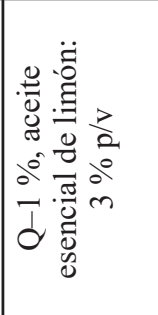 & 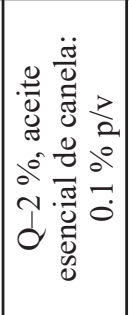 & 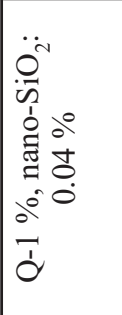 & 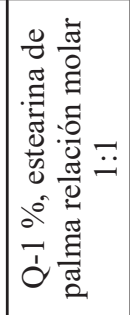 & 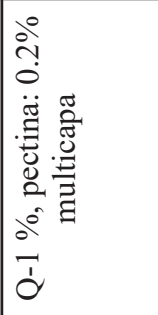 & 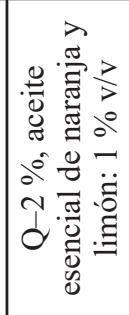 & 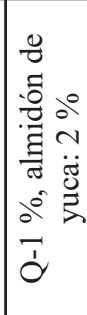 & 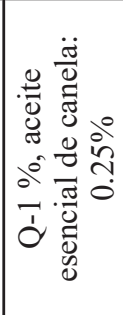 & 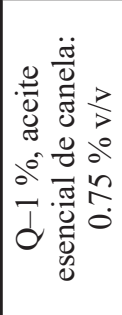 & 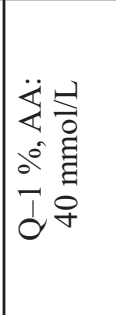 & 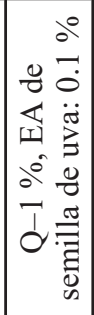 & 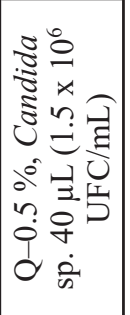 & \\
\hline$\stackrel{巳}{\underline{E}}$ & $\underset{S}{\tilde{S}}$ & 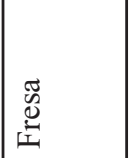 & $\frac{0}{7}$ & 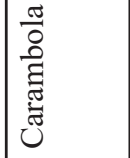 & 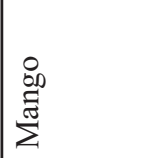 & 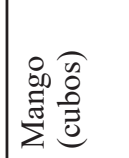 & 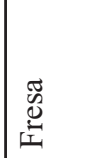 & 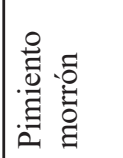 & $=$ & \begin{tabular}{|l}
.$\overline{0}$ \\
$: \frac{1}{3}$
\end{tabular} & 5 & 离 & \\
\hline
\end{tabular}


sin recubrir y/o recubiertos con quitosano sin funcionalizar (Rico et al., 2012). La baja incidencia de enfermedades en los frutos tratados se asocia directamente al control del patógeno causante de la misma por efecto aditivo o sinérgico entre el quitosano y los aceites esenciales (Gutiérrez-Martínez et al., 2017; Rodríguez-Guzmán et al., 2019). La incorporación de aceites esenciales a la matriz del quitosano mejoró significativamente las propiedades de barrera y de intercambio de gases (disminución en la permeabilidad de vapor de agua) (Perdones et al., 2012), lo anterior, atribuido a la naturaleza lipofílica de los aceites esenciales y al incremento del carácter hidrofóbico del recubrimiento, que eventualmente contribuye a retardar la maduración y deshidratación del fruto (Rinaudo, 2008; Mohammadi et al., 2015) sin detrimento en sus atributos sensoriales (sabor, color, apariencia, textura). Únicamente, se han reportado cambios en el sabor de fresas tratadas con el recubrimiento funcionalizado con aceite esencial de limón (Perdones et al., 2012). Adicionalmente, los frutos (pimiento morrón y jujube) recubiertos con quitosano funcionalizado con aceite esencial de canela presentaron una mayor retención de ácido ascórbico, compuestos fenólicos, flavonoides y clorofila en comparación con los frutos sin recubrir o recubiertos con quitosano sin funcionalizar (Xing et al., 2011a, Xing et al. 2011b), estos efectos pueden estar asociados principalmente a una reducción de la difusión del oxígeno entre el fruto y el recubrimiento, lo que ayuda a prevenir la oxidación de estos compuestos (USDA, 2016). El monoterpeno carvacrol, presente en el aceite esencial de algunas especies vegetales como orégano y tomillo (Robledo, Pierini, Díaz-Nieto, Fernández \& Zon, 2019) ha sido utilizado para la funcionalización del quitosano y la conservación de la mora azul $\left(7\right.$ días a $\left.4{ }^{\circ} \mathrm{C}\right)$ con resultados positivos en mantener la calidad del fruto, similares a los ya descritos previamente (Sun, Narciso, Wang, Ference, Bai \& Zhou, 2014). Sin embargo, este tipo de aceite puede presentar efectos fitotóxicos, citotóxicos y genotóxicos a concentraciones entre 0.375 y $3 \mathrm{mmol} / \mathrm{L}$ (de Assis-Alves et al., 2018), por lo que, resulta necesario evaluar la toxicidad potencial del sistema quitosano-carvacrol, cuando este compuesto se utilice como agente funcionalizante de recubrimientos de quitosano para la conservación de alimentos. Por otro lado, no todos los aceites esenciales con los que se puede funcionalizar el quitosano, brindan efectos positivos para la conservación de frutos. Por ejemplo, la funcionalización con aceite esencial de tomillo $(0.1 \%$ $\mathrm{p} / \mathrm{v})$ y clavo $(0.2 \% \mathrm{v} / \mathrm{v})$, no mostró ventajas sobre el control de Rizhopus stolonifer y Penicillium digitatum cuando se aplicó a jitomate y mandarina, respectivamente (Ramos-García et al., 2012; Shao et al., 2015).

Se ha reportado que la actividad antimicrobiana de los recubrimientos de quitosano funcionalizados se debe en gran medida a los grupos aminos libres del quitosano y a los enlaces formados con el aceite esencial, lo que permite al recubrimiento establecer interacciones electrostáticas con la pared externa del microorganismo, lo que resulta en interferencias sobre la síntesis de componentes esenciales para la célula, ya que el quitosano puede actuar como un agente quelante mediante la adsorción de iones metálicos esenciales para las reacciones enzimáticas, además de afectar directamente la estabilidad y permeabilidad (salida de $\mathrm{K}^{+}$y entrada de $\mathrm{Ca}^{+}$) de la pared celular, lo que finalmente resulta en la inactivación o muerte de los microorganismos (Bosquez-Molina, Ronquillo-de Jesús, Bautista-Baños, Verde-Calvo \& Morales-López, 2010; Yuan et al., 2016; Gutiérrez-Martínez et al., 2017; Ramos-Guerrero et al., 2018). Ramos-García et al. (2012) informaron que la combinación de quitosano con aceite esencial de lima y cera de abeja aplicado en jitomate afectó el desarrollo normal de $R$. stolonifer, mediante la alteración de las estructuras reproductivas del hongo, así como daño sobre el micelio e hifas del mismo. Yuan, Chen \& Li (2016) sugieren que el efecto antibacteriano de los recubrimientos de quitosano funcionalizado con aceites esenciales contra microorganismos aerobios, pudiera incrementarse debido al bajo contenido de oxígeno entre el fruto y el recubrimiento.

Es importante resaltar que la efectividad de este tipo de recubrimientos sobre el control de los microorganismos estará en función de la pureza, grado de desacetilación y fuente de obtención del quitosano, además de la susceptibilidad de los microorganismos que se desee controlar, así como de la relación quitosano: aceite esencial, velocidad de liberación y disponibilidad de los aceites (Yuan et al., 2016; GutiérrezMartínez et al., 2017).

\section{FUNCIONALIZACIÓN CON EXTRACTOS NATURALES,} ÁCIDO ASCÓRBICO Y ÁCIDO CLOROGÉNICO

Los recubrimientos de quitosano también han sido funcionalizados con extractos acuoso-orgánicos de diferentes especies vegetales, como son Moringa oleifera, flores de Paeonnia rockii, hojas de arándano y semillas de uva para la conservación de las fresas $\left(16\right.$ días a $\left.4{ }^{\circ} \mathrm{C}\right)$, arándanos (35 días a $2{ }^{\circ} \mathrm{C}$ ) y uva roja $\left(28\right.$ días a $\left.0-1{ }^{\circ} \mathrm{C}\right)$, respectivamente (Xu et al., 2007; Yang et al., 2014; Pagliarulo et al., 2016; MaderaSantana et al., 2019). Algunas ventajas particulares han sido reportadas para cada uno de estos recubrimientos. Por ejemplo, los frutos tratados con recubrimientos funcionalizados con extracto de flores de Paeonnia rockii y hojas de arándano exhibieron cambios mínimos sobre su calidad en general, así como mayor retención de ácido ascórbico y compuestos fenólicos en comparación con los frutos recubiertos con quitosano sin funcionalizar (Yang et al., 2014; Pagliarulo et al., 2016), lo anterior, sin modificar los atributos sensoriales de los frutos tratados (Xu et al., 2007; Pagliarulo et al., 2016). Por su parte, la aplicación de un recubrimiento de quitosano funcionalizado con extracto de Moringa oleifera mejora el índice de sabor y dulzura de las fresas, pero no los parámetros de calidad en comparación con el grupo control (Madera-Santana et al., 2019). En general, la composición de los extractos naturales que se agregan al quitosano, están fuertemente representados 
por compuestos con capacidad antioxidante demostrada (ácido ascórbico, compuestos fenólicos y flavonoides). Estos antioxidantes pueden reducir el ataque de microorganismos, disminuir reacciones de oscurecimiento en la cáscara de los frutos y disminuir el estrés oxidativo sobre todo en frutos precortados. Asimismo, su combinación con el quitosano puede cambiar la estructura del recubrimiento y reducir la entrada del oxígeno disminuyendo la tasa de respiración y producción de etileno, todo eso conlleva a retardar la maduración del fruto (TorresAguirre et al., 2018; Yuan et al., 2016). Adicionalmente, se ha reportado que el quitosano puede exhibir actividad antioxidante debido a la presencia de los grupos aminos libres, sin embargo, este efecto depende directamente de la concentración, peso molecular y grado de desacetilación del biopolímero (Kim \& Thomas, 2007).

Liu, Yuan, Chen, Li \& Liu (2014) demostraron que la aplicación del recubrimiento de quitosano con ácido ascórbico tuvo un efecto positivo sobre la firmeza y color de los frutos de ciruela durante 20 días a $5{ }^{\circ} \mathrm{C}$, además de una disminución en la tasa de respiración en comparación con los frutos tratados individualmente con los mismos componentes. $\mathrm{La}$ retención de la firmeza fue asociada al decremento en las actividades enzimáticas de las enzimas pectin metil esterasa y poligalacturonasa. Por su parte, Sun, Liang, Xie, Lei and Mo (2010) reportaron una disminución en la actividad enzimática de la polifenol oxidasa y peroxidasa en frutos de lichi recubiertos con quitosano funcionalizado y ácido ascórbico, lo que ayudó a conservar la calidad del fruto durante su almacenamiento (30 días a $\left.4^{\circ} \mathrm{C}\right)$.

Por su parte, Jiao et al. (2019) evaluaron el efecto de la incorporación de ácido clorogénico en una matriz de quitosano y su efecto sobre la vida de anaquel en duraznos. Los autores reportaron que los parámetros de calidad (pérdida de peso, retraso de senescencia y tasa de respiración, sólidos solubles totales, acidez titulable, $\mathrm{pH}$, retención de firmeza y ácido ascórbico) del fruto recubierto se mantuvieron adecuados durante 8 días a $20{ }^{\circ} \mathrm{C}$. Estos efectos fueron atribuidos al efecto sinérgico entre el ácido clorogénico y el quitosano, así como a la disminución en la difusión de oxígeno entre el fruto y el recubrimiento.

\section{FUNCIONALIZACIÓN CON COMPUESTOS DE ORIGEN PROTEICO}

Recientemente, diversos extractos proteicos (soya, quínoa, lactosuero y caroteno-proteínas) han sido aprovechados para funcionalizar al quitosano mediante la incorporación y homogenización de ambos componentes (Maccora et al., 2019), con el objetivo de prolongar la vida de anaquel del albaricoque $\left(42\right.$ días a $4{ }^{\circ} \mathrm{C}$ ), fresas $\left(9-11\right.$ días a $4{ }^{\circ} \mathrm{C}$ ) y plátanos (9 días a temperatura ambiente) (Valenzuela et al., 2015; Arce-Ortiz, Ortega-Villalba, Ochoa-Martínez \& VélezPasos, 2016; Zhang, Chen, Lai, Wang \& Yang, 2018; Hajji et al., 2018). Los frutos tratados con los recubrimientos de quitosano con aislado de proteína de soya (albaricoque), quinoa y carotenoproteínas (fresas) mantuvieron estables los parámetros de calidad, principalmente la textura. Los efectos observados en albaricoque, se atribuyen a la inhibición de la degradación de la pectina presente en la pared celular del fruto (Zhang et al., 2018), mientras que los efectos observados en las fresas se asocian a la disminución en la emisión de $\mathrm{CO}_{2}$, que fue menor en comparación con frutos recubiertos sólo con quitosano (Hajji et al., 2018; Valenzuela et al., 2015). Adicionalmente, el uso de un recubrimiento funcionalizado con proteína de lacto suero retrasó (2 días) la aparición del pico climatérico en plátano en comparación con el fruto control (Arce et al., 2016). Di Pierro et al. (2006) mencionan que al funcionalizar el quitosano con matrices proteicas puede existir un mayor entrecruzamiento en la matriz, lo que mejora las propiedades mecánicas (esfuerzo a la tensión de 26.2 MPa) y de barrera (permeabilidad al oxígeno, $\mathrm{CO}_{2}$ y vapor de agua de 7.8, 8.3 y $0.88 \mathrm{~cm}^{3} \mu \mathrm{m} / \mathrm{m}^{2}$ día $\mathrm{kPa}$, respectivamente) del recubrimiento resultante, en comparación con el recubrimiento de quitosano sin funcionalizar (esfuerzo a la tensión de 14.4 MPa, permeabilidad al oxígeno, $\mathrm{CO}_{2}$ y vapor de agua de 20.6, 20.7 y $3.24 \mathrm{~cm}^{3}$ $\mu \mathrm{m} / \mathrm{m}^{2}$ día $\mathrm{kPa}$, respectivamente). Es importante señalar que los resultados asociados al uso de aislados proteicos para funcionalizar quitosano, pueden optimizarse mediante cambios en las proporciones de cada componente del recubrimiento funcionalizado (Hajji et al., 2018).

\section{FunCIONALIZACIÓN CON POLISACÁRIDOS}

Una alternativa viable para la funcionalización del quitosano es la adición de otros polisacáridos como la pectina, carboximetilcelulosa y almidón de yuca (Campos, Kwiatkowski \& Clemente, 2011; Medeiros, Pinheiro, Carneiro-da-Cunha \& Vicente, 2012; Gol et al. 2013), con lo que se mejoran las propiedades de barrera del recubrimiento (reducción en la permeabilidad de oxígeno y vapor de agua) y extiende la vida de anaquel de frutas y hortalizas con cambios mínimos en los parámetros de calidad (baja pérdida de peso con cambios mínimos en $\mathrm{pH}$, acidez titulable y sólidos solubles totales), mayor retención de ácido ascórbico, compuestos fenólicos y antocianinas, además de mejorar su apariencia y brindarles protección antimicrobiana (Campos et al., 2011; Medeiros et al., 2012; Gol et al., 2013). Por otro lado, se han reportado efectos negativos sobre la calidad de los nectarines $\left(4^{\circ} \mathrm{C}, 95 \% \mathrm{HR}, 9\right.$ días) al aplicar un recubrimiento de quitosano combinado con alginato de sodio, lo anterior, debido principalmente a la alta solubilidad y permeabilidad del vapor de agua del alginato de sodio, lo que rompe el equilibrio de la "atmósfera modificada" creada entre el fruto y el recubrimiento (Chiabrando \& Giacalone, 2016). Aunque la funcionalización del quitosano con otros polisacáridos es una opción viable, deben considerarse factores como la solubilidad y estabilidad del biopolímero funcionalizante para obtener mejores resultados (Bautista-Baños et al., 2017; Rinaudo, 2014). 


\section{FUNCIONALIZACIÓN CON AGENTES BIOLÓGICOS}

La funcionalización del quitosano con agentes biológicos, principalmente levaduras y lactobacilos, es otra alternativa prometedora para minimizar las pérdidas postcosecha de frutas y hortalizas (Rojas-Grau, Soliva-Fortuny \& Martín-Belloso, 2009). Chantrasri, Sardsud, Sangchote and Sardsud (2007) reportaron que la incorporación de Candida sp. al recubrimiento a base de quitosano minimizó la incidencia de antracnosis (hasta $94 \%$ ) causada por C. gloeosporioides en mango (7 días a $25^{\circ} \mathrm{C}$ ). De manera similar, Wang et al. (2019) reportaron que la funcionalización del quitosano con Cryptococcus laurentii redujo la incidencia del moho azul causado por $P$. italicum en frutos de uva $\left(8\right.$ días a $\left.20{ }^{\circ} \mathrm{C}\right)$. Además, a la matriz formada con quitosano y un agente biológico se le puede incorporar compuestos previamente encapsulados, como el ácido ascórbico, compuestos fenólicos y aceites esenciales que se liberan gradualmente bajo condiciones específicas, con el objetivo de mejorar las propiedades tecnológicas (mecánicas, fisicoquímicas, térmicas y de barrera) y funcionales (actividad antimicrobiana y antioxidante) del recubrimiento resultante(Yin et al., 2019). Sin embargo, aún se requieren más estudios para encontrar las condiciones adecuadas que permitan incrementar la vida de anaquel de los frutos tratados con este tipo de recubrimiento, sin afectar los atributos de calidad de los mismos, además de garantizar la viabilidad de los microorganismos empleados durante la funcionalización (Castillo-Escandón, Fernández-Michel, Cueto-Wong \& Montfort, 2019).

\section{FUNCIONALIZACIÓN CON COMPUESTOS INORGÁNICOS}

Compuestos inorgánicos como el dióxido de silicio ( $\mathrm{Yu}$ et al., 2012; Shi et al., 2013) y titanio (Kaewklin et al., 2018), montmorillonita (Xu, Qin \& Ren, 2018) y plata (Ortiz-Duarte, Pérez-Cabrera,Artés-Hernández \& Martínez-Hernández, 2019) se han utilizado para la funcionalización del quitosano con el objetivo de prolongar la vida de anaquel del fruto de jujube (32 días a temperatura ambiente), longan (8 días a temperatura ambiente), jitomate $\left(15\right.$ días a $\left.20^{\circ} \mathrm{C}\right)$, mandarina $\left(11\right.$ días a $\left.10^{\circ} \mathrm{C}\right)$ y melón ( 13 días a $\left.5^{\circ} \mathrm{C}\right)$, respectivamente; con modificaciones mínimas sobre parámetros de calidad y atributos sensoriales de los frutos en comparación con los controles recubiertos con quitosano sin funcionalizar. Se ha reportado además, que el recubrimiento de quitosano funcionalizado con $\mathrm{TiO}$, tiene la capacidad de degradar el etileno en presencia de luz UV (320 $390 \mathrm{~nm}$ durante $180 \mathrm{~min}$ ), debido a que el $\mathrm{TiO}_{2}$ tiene la capacidad de generar radicales $\bullet \mathrm{OH}$ y especies reactivas de oxígeno en presencia de luz UV, los cuales a su vez pueden reaccionar con moléculas orgánicas como el etileno y descomponerlo en $\mathrm{CO}_{2}$ y agua, lo que ayuda a retardar el proceso de maduración de los frutos de jitomate (Kaewklin et al., 2018).

\section{FUNCIONALIZACIÓN CON OTROS COMPUESTOS}

Se ha reportado que la funcionalización del quitosano con $\beta$-ciclodextrina extiende la vida de anaquel de uvas rojas
(12 días a $4{ }^{\circ} \mathrm{C}$ ) (Youwei \& Yinzhe, 2013), mientras que, la funcionalización con clorhidrato prolonga la vida de anaquel de la lechuga ( 7 días) y kiwi (20 a 25 días, $4{ }^{\circ} \mathrm{C}$ ), con una disminución en la incidencia del moho gris causado por $B$. cinerea y Pectobacterium carotovorum, respectivamente (Fortunati et al., 2017). De igual manera, Li, Ye, Hou \& Zhang (2018) lograron extender la vida de anaquel (25 días a $10{ }^{\circ} \mathrm{C}$ ) de frutos de mandarina tratados con un recubrimiento funcionalizado con $\xi$-polilisina, sin cambios en sus atributos de calidad y mayor retención de ácido ascórbico. Por su parte, la funcionalización con estearina de palma ha sido efectiva para prolongar la vida de anaquel de los frutos de carambola (20 días a temperatura ambiente) asociado a la reducción en la tasa de respiración y producción de etileno (Nurul-Hanani, Halimahton-Zahrah \& Zaibunnisa, 2012). Resultados similares se reportaron al aplicar este tipo de recubrimiento (quitosanoestearina de palma) en guayaba (31 días a $5^{\circ} \mathrm{C}$ ), además de una disminución en el daño por frío y pérdida de peso en comparación con los frutos recubiertos sólo con quitosano o frutos sin recubrir (Nurul-Hanani, Halimahton-Zahrah \& Zaibunnisa, 2016). Finalmente, la funcionalización con ácido salicílico ha sido efectiva para minimizar el daño por frío (12 días a $\left.2{ }^{\circ} \mathrm{C}\right)$ y pérdida de peso $(<3 \%)$ en pepino, además de favorecer la retención de ácido ascórbico y clorofila (Zhang et al., 2015b). Estos efectos se deben a que el ácido salicílico actúa como agente inductor para la activación del sistema de defensa del fruto, entre ellos la síntesis de proteínas de choque térmico, las cuales son proteínas que protegen a otras proteínas presentes en la membrana de la célula y evitan su desnaturalización, lo que retarda el deterioro de la membrana celular y pérdida de agua (Rasouli, Saba \& Ramezanian, 2019).

\section{CONCLUSIONES}

La funcionalización de recubrimientos comestibles mediante la incorporación de diversos compuestos es una alternativa viable para mejorar las propiedades tecnológicas y funcionales de los recubrimientos comestibles convencionales. El uso de recubrimientos a base de quitosano funcionalizado puede prevenir o minimizar la incidencia de enfermedades postcosecha, además de retrasar el deterioro natural de varios productos hortofrutícolas, conservar su calidad y aumentar su vida útil. Sin embargo, se requieren más estudios para mejorar las condiciones de obtención y aplicación de este tipo de recubrimientos comestibles en frutas y hortalizas, además de complementar la caracterización de sus propiedades fisicoquímicas, mecánicas y térmicas que pudieran condicionar el desempeño de los mismos tanto in vitro como in vivo.

\section{Agradecimientos}

Anaya-Esparza Luis M. agradece al Consejo Nacional de Ciencia y Tecnología (CONACYT) por la beca otorgada (702634) para estudios de posgrado. 


\section{REFERENCIAS}

Arce-Ortiz, K. L., Ortega-Villalba, K. J., Ochoa-Martínez, C. I. \& Vélez-Pasos, C. (2016). Evaluation of the water vapor permeability for whey protein/chitosan films and its effect on respiration of coated banana. INNOTEC, 11(1), 59-64.

Bautista-Baños, S., Ventura-Aguilar, R. I., Correa-Pacheco, Z. \& Corona-Rangel, M. L. (2017). Chitosan: a versatile antimicrobial polysaccharide for fruit and vegetables in postharvest-a review. Revista Chapingo Serie Horticultura, 23(2), 103-121. DOI: http://dx.doi.org/10.5154/r. rchsh.2016.11.030

Berumen-Varela, G., Coronado Partida, L. D., Ochoa Jiménez, V. A., Chacón López, M. A. \& Gutiérrez Martínez, P. (2015). Effect of chitosan on the induction of disease resistance against Colletotrichum sp. in mango (Mangifera indica L.) cv. Tommy Atkins. Investigación y Ciencia de la Universidad Autónoma de Aguascalientes, 66(1), 16-21.

Bilalis, P., Katsigiannopoulos, D., Avgeropoulos, A. \& Sakellariou, G. (2014). Non-covalent functionalization of carbon nanotubes with polymers. RCS Advances, 4, 2911-2934. DOI: 10.1039/C3RA44906H

Bosquez-Molina, E., Ronquillo-de Jesús, E., Bautista-Baños, S., Verde-Calvo, J. R. \& Morales-López, J. (2010). Evaluation of the inhibitory effect of essential oils against Colletotrichum gloesoporioides and Rhizopus stolonifer in stored papaya fruit and their possible application in coatings. Postharvest Biology and Technology, 57, 132-137. DOI: https://doi.org/10.1016/j.postharvbio.2010.03.008

Campos, R. P., Kwiatkowski, A. \& Clemente, E. (2011). Post-harvest conservation of organic strawberries coated with cassava starch and chitosan. Revista Ceres, 58(5), 554-560. DOI: http://dx.doi.org/10.1590/S0034737X2011000500004

Castillo-Escandón, V., Fernández-Michel, S. G., Cueto-Wong, M. C. \& Montfort, G. R. C. (2019). Criterios y estrategias tecnológicas para la incorporación y supervivencia de probióticos en frutas, cereales y sus derivados. TIP Revista Especializada en Ciencias Químico-Biológicas, 22(1), 1-17. DOI: https://doi.org/10.22201/fesz.23958723e.2019.0.173

Chantrasri, P., Sardsud, V., Sangchote, S. \& Sardsud, U. (2007). Combining yeasts and chitosan treatment to reduce anthracnose fruit rot in mangoes. Asian Journal of Biology Education, 3(1), 40-46.

Chen, Y. C., Wang, C. H., Lai, L. S. \& Lin, K. W. (2003). Rheological properties of chitosan and its interaction with porcine myofibrillar proteins as influenced by chitosan's degree of deacetylation and concentration. Journal of Food Science: Food Chemistry and Toxicology, 68(3), 826-831. DOI: https://doi.org/10.1111/j.1365-2621.2003.tb08250.x

Chiabrando, V. \& Giacalone, G. (2016). Effect of chitosan and sodium alginate edible coatings on the postharvest quality of fresh-cut nectarines during storage. Fruits, 71(2), 79-85. DOI: $10.1051 /$ fruits/2015049 de Assis-Alves, T., Fontes-Pinheiro, P., Praca-Fontes, M. M., Andrade-Vieira, L. F., Barelo-Correa, K., de Assis-Alves, T., Aparecida da Cruz, F., Lacerda-Junior, V., Ferreira, A. \& Bastos-Soares, T. C. (2018). Toxicity of thymol, carvacrol and their respective phenoxyacetic acids in Lactuca sativa and Sorghum bicolor. Industrial Crops and Products, 114, 59-67. DOI: https://doi.org/10.1016/j. indcrop.2018.01.071

Di Pierro, P., Chico, B., Villalonga, R., Mariniello, L., Damiao, A. E., Masi, P. \& Pota, R. (2006). Chitosan-whey protein edible films produced in the absence or presence of transglutaminase: Analyisis of their mechanical and barrier properties. Biomacromolecules, 7(1), 744-749. DOI: $10.1021 / \mathrm{bm} 050661 \mathrm{u}$

Fortunati, E., Giovanale, G., Luzi, F., Mazzaglia, A., Kenny, J. M., Torre, L. \& Balestra, G. M. (2017). Effective postharvest preservation of kiwifruit and romaine lettuce with a chitosan hydrochloride coating. Coatings, 7, 2-15. DOI: https://doi. org/10.3390/coatings7110196

Gol, N. B., Patel, P. R. \& Ramana-Rao, T. V.(2013). Improvement of quality and shelf-life of strawberries with edible coatings enriched with chitosan. Postharvest Biology and Technology, 85(1), 185-195. DOI: https://doi.org/10.1016/j. postharvbio.2013.06.008

Gutiérrez-Martínez, P., Bautista-Baños, S., Berúmen-Varela, G., Ramos-Guerrero, A. \& Hernández-Ibáñez, A. M. (2017). In vitro response of Colletotrichum to chitosan. in vitro response of Colletotrichum to chitosan. Effect on incidence and quality on tropical fruit. Enzymatic expression in mango. Acta Agronomica, 66(2), 282-289. DOI: http:// dx.doi.org/10.15446/acag.v66n2.53770

Hajji, S., Younes, I., Affes, S., Boufi, S. \& Nasri, M. (2018). Optimization of the formulation of chitosan edible coatings supplemented with carotenoproteins and their use for extending strawberries postharvest life. Food Hydrocolloids, 83(1), 375-392. DOI: https://doi. org/10.1016/j.foodhyd.2018.05.013

Jiao, W., Shu, C., Li, X., Cao, J., Fan, X. \& Jiang, W. (2019). Preparation of a chitosan-chlorogenic acid conjugate and its application as edible coating in postharvest preservation of peach fruit. Postharvest Biology and Technology, 154(1), 129-136. DOI: https://doi.org/10.1016/j. postharvbio.2019.05.003

Kaewklin, P., Siripatrawan, U., Suwanagul, A. \& Lee, Y.S. (2018). Active packaging from chitosan-titanium dioxide nanocomposite film for prolonging storage life of tomato fruit. International Journal of Biological Macromolecules, 112(1), 523-529. DOI: https://doi.org/10.1016/j. ijbiomac.2018.01.124

Kim, K.W. \& Thomas, R. L. (2007). Antioxidative activity of chitosan with varying molecular weights. Food Chemistry, 101, 308-313. DOI: 10.1016/j.foodchem.2006.01.038

Layek, R. K. \& Nandi, A. K. (2013). A review on synthesis and properties of polymer functionalized graphene. 
Polymer, 54, 5087-5103. DOI: https://doi.org/10.1016/j. polymer.2013.06.027

Li, N. Y., Ye, Q. Q., Hou, W. F. \& Zhang, G. Q. (2018). Development of antibacterial \&-polylysine/chitosan hybrid films and the effect on citrus. International Journal of Biological Macromolecules, 118, 2051-2056. DOI: 10.1016/j.ijbiomac.2018.07.074

Liu, K., Yuan, C., Chen, Y., Li, H. \& Liu, J. (2014). Combined effects of ascorbic acid and chitosan on the quality maintenance and shelf life of plums. Scientia Horticulturae, 176, 45-53. DOI: https://doi.org/10.1016/j. scienta.2014.06.027

López, A., Rivas, J., Loaiza, M. \& Sabino, M. (2010). Degradación de películas plastificadas de quitosano obtenidas a partir de conchas de camarón. Revista de la Facultad de Ingeniería UCV, 25, 133-143.

López-Mata, M. A., Ruiz-Cruz, S., Navarro-Preciado, C., Ornelas-Paz, J. J., Estrada-Alvarado, M. I. \& GassosOrtega, L. E. (2012). Effect of chitosan edible coatings in the microbial reduction and conservation of the quality of strawberries. Revista de Ciencias Biológicas y de la Salud, 14(1), 33-43.

Maccora, D., Dini, V., Battocchio, C., Fratoddi, I., Cartoni, A., Rotili, D., Castagnola, M., Faccini, R., Bruno, I., Scotognella, T., Giordano, A. \& Venditti, I. (2019). Gold nanoparticles and nanorods in nuclear medicine: A mini review. Applied Sciences, 9, 3232. DOI: 10.3390/app9163232

Madera-Santana, J. T., De Dios-Aguilar, M. A., Colín-Chávez, C., Mariscal-Amaro, L. A., Nuñez-Colín, C. A., VelozGarcía, R., Gizmán-Maldonado, S. H., Peña-Caballero, V., Grijalva-Verdugo, C. P. \& Rodríguez-Núñez, J. R. (2019). Coating based on chitosan and aqueous extract of Moringa oleifera leaf obtained by UMAE and its effect on the physicochemical properties of strawberry (Fragariax ananassa). Revista de Ciencias Biológicas y de la Salud, 21(2), 155-163.

Medeiros, B. G. S., Pinheiro,A. C., Carneiro-da-Cunha, M. G. \& Vicente,A.A.(2012). Development and characterization of a nanomultilayer coating of pectin and chitosan-Evaluation of its gas barrier properties and application on 'Tommy Atkins' mangoes. Journal of Food Engineering, 110(3), 457-464. DOI: https://doi.org/10.1016/j.jfoodeng.2011.12.021

Mohammadi, A., Hashemi, M. \& Hosseini, S. M. (2015). Chitosan nanoparticles loaded with Cinnamomum zeylanicum essential oil enhance the shelf life of cucumber during cold storage. Postharvest Biology and Technology, 110(1), 203-213. DOI: https://doi.org/10.1016/j. postharvbio.2015.08.019

Mohandas, A., Deepthi, S., Biswas, R. \& Jayakumar, R. (2017). Chitosan based metallic nanocomposite scaffolds as antimicrobial wound dressings. Bioactive Materials, 3, 267277.DOI: https://doi.org/10.1016/j.bioactmat.2017.11.003 Mujtaba, M., Mosri, R. E., Kerch, G., Elsabee, M. Z., Kaya, M., Labidi, J. \& Khawar, K. M. (2019). Current advancements in chitosan-based film production for food technology: A review. International Journal of Biological Macromolecules, 121(1), 889-904. DOI: https://doi. org/10.1016/j.ijbiomac.2018.10.109

Nurul-Hanani, M.Z., Halimahton-Zahrah, M. S. \& Zaibunnisa, A. H. (2012). Effect of chitosan-palm stearin edible coating on the postharvest life of star fruits (Averrhoa carambola L.) stored at room temperature. International Food Research Journal, 19(4), 1433-1438.

Nurul-Hanani, M.Z., Halimahton-Zahrah, M. S. \& Zaibunnisa, A. H. (2016). Effect of edible lipid-chitosan film forming dispersion on postharvest life of guava stored at chilled temperature. Malasyan Journal of Analytical Sciences, 20(3), 618-625. DOI: http://dx.doi.org/10.17576/mjas2016-2003-22

Ortiz-Duarte, G., Pérez-Cabrera, L. E., Artés-Hernández, F., \& Martínez-Hernández, G. B. (2019). Ag-chitosan nanocomposites in edible coatings affect the quality of fresh-cut melon. Postharvest Biology and Technology, 147(1), 174-184. DOI: https://doi.org/10.1016/j. postharvbio.2018.09.021

Pagliarulo, C., Sansone, F., Moccia, S., Russo, G. L., Aquino, R. P., Salvatore, P., Di Stasio, M. \& Volpe, M. G. (2016). Preservation of strawberries with an antifungal edible coating using peony extracts in chitosan. Food and Bioprocess Technology, 9(11), 1951-1960. DOI: https:// doi.org/10.1007/s11947-016-1779-x

Perdones, A., Sánchez-González, L. \& Vargas, M. (2012). Effect of chitosan-lemon essential oil coatings on storagekeeping quality of strawberry. Postharvest Biology and Technology, 70(1), 32-41. DOI: https://doi.org/10.1016/j. postharvbio.2012.04.002

Porat, R., Lichter, A., Terry, L. A., Harker, R. \& Buzby, J. (2018). Postharvest losses of fruit and vegetables during retail and it consumers' homes: Quantifications, causes, and means of prevention. Postharvest Biology and Technology, 139, 135-149. DOI: https://doi.org/10.1016/j. postharvbio.2017.11.019

Ramos-García, M., Bosquez-Molina, E., Hernández-Romano, J., Zavala-Padilla, G., Térres-Rojas, E., Alia-Tejacal, I., Barrera-Necha, L., Hernández-López, M. \& BautistaBaños, S. (2012). Use of chitosan-based edible coatings in combination with other natural compounds, to control Rhizopus stolonifer and Escherichia coli DH5 $\alpha$ in fresh tomatoes. Crop Protection, 38(1), 1-6. DOI: https://doi. org/10.1016/j.cropro.2012.02.016

Ramos-Guerrero, A., González-Estrada, R., Hanako-Rosas, G., Bautista-Baños, S., Acevedo-Hernández, G., Tiznado-Hernández, M. E. \& Gutiérrez-Martínez, P. (2018). Use of inductors in the control of Colletotrichum gloeosporioides and Rhizopus stolonifer isolated from soursop fruits: in vitro tests. Food Science and Biotechnology, 27(3), 755-763. DOI: https://doi. org/10.1007/s10068-018-0305-5 
Rasouli, M., Saba, M. K. \& Ramezanian, A. (2019). Inhibitory effect of salicylic acid and Aloe vera gel edible coating on microbial load and chilling injury of orange fruit. Scientia Horticulturae, 247, 27-34. DOI: https://doi.org/10.1016/j. scienta.2018.12.004

Rico, F., Gutiérrez, C.\& Díaz-Moreno, C. (2012). Effect of edible coating of chitosan and essential oils on microbiological quality of minimally processed mango (Mangifera indica L.). Vitae, 19 (1), S117-S119.

Rinaudo, M. (2008). Behaviour of amphiphilic polysaccharides in aqueous medium. TIP Revista Especializada en Ciencias Químico-Biológicas, 11(1), 35-40.

Rinaudo, M. (2014). Biomaterials based on a natural polysaccharide: alginate. TIP Revista Especializada en Ciencias Químico-Biológicas, 17(1), 92-96. DOI: https:// doi.org/10.1016/S1405-888X(14)70322-5

Robledo, S. N., Pierini, G. D., Díaz-Nieto, C.H., Fernández, H., \& Zon, M. A. (2019). Development of an electrochemical method to determine phenolic monoterpenes in essential oils. Talanta, 196,362-369. DOI: https://doi.org/10.1016/j. talanta.2018.12.069

Rodríguez-Guzmán, C. A., González-Estrada, R. R., BautistaBaños, S. \& Gutiérrez-Martínez, P. (2019). Efecto del quitosano en el control de Alternaria sp. en plantas de jitomate en invernadero. TIP Revista Especializada en Ciencias Químico-Biológicas, 22, 1-7. DOI: 10.22201/ fesz.23958723e

Rojas-Grau, M. A., Soliva-Fortuny, R. \& Martín-Belloso, O. (2009). Edible coatings to incorporate active ingredients to fresh-cut fruits: a review. Trends in Food Science \& Technology, 20(10), 438-447. DOI: https://doi. org/10.1016/j.tifs.2009.05.002

Romanazzi, G., Sanzani, S. M., Bi, Y., Tian, S., GutiérrezMartínez, P. \& Alkan, N. (2016). Induced resistance to control postharvest decay of fruit and vegetables. Postharvest Biology and Technology, 122, 82-94.

Shao, X., Cao, B., Xu, F., Xie, S., Yu, D. \& Wang, H. (2015). Effect of postharvest application of chitosan combined with clove oil against citrus green mold. Postharvest Biology and Technology, 99(1), 34-43. DOI: https://doi.org/10.1016/j. postharvbio.2014.07.014

Shi, S., Wang, W., Liu, L., Wu, S., Wei, Y. \& Li, W. (2013). Effect of chitosan/nano-silica coating on the physicochemical characteristics of longan fruit under ambient temperature. Journal of Food Engineering, 118(1), 125-131. DOI: https:// doi.org/10.1016/j.jfoodeng.2013.03.029

Solano-Doblado, L. G., Alamilla-Beltrán, L. \& JiménezMartínez, C. (2018). Películas y recubrimientos comestibles funcionalizados. TIP Revista Especializada en Ciencias Químico-Biológicas, 21(2), 30-42. DOI: https://doi. org/10.22201/fesz.23958723e.2018.0.153

Sun, D., Liang, G., Xie, J., Lei, X. \& Mo, Y. (2010). Improved preservation effects of litchi fruit by combining chitosan coating with ascorbic acid treatment during postharvest storage. African Journal of Biotechnology, 9 (22), 3272-3279.

Sun, X., Narciso, J., Wang, Z., Ference, C., Bai, J. \& Zhou, K. (2014). Effects of chitosan-essential oil coatings on safety and quality of fresh blueberries. Journal of food science, 79(5), 955-960. DOI: 10.1111/1750-3841.12447

Torres-Aguirre, G. A., Muñoz-Bernal, O. A., ÁlvarezParrilla, E., Nuñez-Gastelúm, J. A., Wall-Medrano, A., Sáyago-Ayerdi, S. G. \& de la Rosa, L. A. (2018). Optimización de la extracción e identificación de compuestos polifenólicos en anís (Pimpinella anisum), clavo (Syzygium aromaticum) y cilantro (Coriandrum sativum) mediante HPLC acoplado a espectrometría de masas. TIP Revista Especializada en Ciencias QuímicoBiológicas, 21(2), 103-115. DOI: https://doi.org/10.22201/ fesz.23958723e.2018.2.137

USDA. (2016). United States Department of Agriculture. The commercial storage of fruits, vegetables, and florist and nursery stocks. En K. C. Gross, C. Y. Wang, \& M. Saltveit (Eds.), Agriculture Handbook (pp. 11-166). California, USDA. https:/www.ars.usda.gov/ARSUserFiles/oc/np/ CommercialStorage/CommercialStorage.pdf

Valenzuela, C., Tapia, C., Lopéz, L., Bunger, A., Escalona, V. \& Abugoch, L. (2015). Effect of edible quinoa protein-chitosan based films on refrigerated strawberry (Fragaria $\times$ ananassa) quality. Electronic Journal of Biotechnology, 18(6), 406-411. DOI: https://doi. org/10.1016/j.ejbt.2015.09.001

Wang, F., Deng, J., jiao, J., Lu, Y., Yang, L. \& Shi, Z. (2019). The combined effects of carboxymethyl-chitosan and Cryptococcus laurentii treatment on postharvest blue mold caused by Penicillium italicum in grapefruit fruit. Scientia Horticulturae, 253(1), 35-41. DOI: https://doi. org/10.1016/j.scienta.2019.04.031

Wu, C., Wang, L., Fang, Z., Hu, Y., Chen, S., Sugawara, T. $\&$ Ye. X. (2016). The effect of the molecular architecture on the antioxidant properties of chitosan gallate. Marine Drugs, 14(5), 95. DOI: 10.3390/md14050095

Xing, Y., Li, X., Xu, Q., Yun, J., Lu, Y. \& Tang, Y. (2011a). Effects of chitosan coating enriched with cinnamon oil on qualitative properties of sweet pepper (Capsicum annuum L.). Food Chemistry, 124(4), 1443-1450. DOI: https://doi. org/10.1016/j.foodchem.2010.07.105

Xing, Y., Xu, Q., Che, Z., Li, X. \& Li, W. (2011b). Effects of chitosan-oil coating on blue mold disease and quality attributes of jujube fruits. Food \& Fucntion, 2(8), 446-474. DOI: $10.1039 / \mathrm{c} 1$ fo10073d

Xoca-Orozco, L. A., Cuellar-Torres, E. A., González-Morales, S., Gutiérrez-Martínez, P., López-García, U., HerreraEstraella, L., Vega-Arreguín, J. \& Chacón-López, A. (2017). Transcriptomic analysis of avocado Hass (Persea americana Mill) in the interaction system fruit-chitosancolletotrichum, Frontiers in Plant Science, 8, 956. DOI: 10.3389/fpls.2017.00956 
Xu, D., Qin, H. \& Ren, D. (2018). Prolonged preservation of tangerine fruits using chitosan/montmorillonite composite coating. Postharvest Biology and Technology, 143(1), 50-57. DOI: https://doi.org/10.1016/j. postharvbio.2018.04.013

Xu, W. T., Huang, K. L., Guo, F., Qu, W., Yang, J. J., Liang, Z. H. \& Luo, Y.B. (2007). Postharvest grapefruit seed extract and chitosan treatments of table grapes to control Botrytis cinerea. Postharvest Biology and Technology, 46(1), 86-94. DOI: https://doi.org/10.1016/j.postharvbio.2007.03.019

Yang, G., Yue, J., Gong, X., Qian, B., Wang, H., Deng, Y. \& Zhao, Y. (2014). Blueberry leaf extracts incorporated chitosan coatings for preserving postharvest quality of fresh blueberries. Postharvest Biology and Technology, 92(1), 4653.DOI: https://doi.org/10.1016/j.postharvbio.2014.01.018

Yin, C., Huang, C., Wang, J., Liu, Y., Lu, P. \& Huang, L. (2019). Effect of chitosan- and alginate-based coatings enriched with cinnamon essential oil microcapsules to improve the postharvest quality of mangoes. Materials, 12, 2039. DOI: https://doi.org/10.3390/ma12132039

Youwei, Y. \& Yinzhe, R. (2013). Grape preservation using chitosan combined with B-cyclodextrin. International Journal of Agronomy, ID: 209235, 1-8. DOI: http://dx.doi. org/10.1155/2013/209235
Yuan, G., Chen, X. \& Li, D. (2016). Chitosan films and coatings containing essential oils: The antioxidant and antimicrobial activity, and application in food systems. Food Research International, 89(1), 117-128. DOI: https:// doi.org/10.1016/j.foodres.2016.10.004

Yu, Y., Zhang, S., Ren, Y., Li, H., Zhang, X. \& Di, J. (2012). Jujube preservation using chitosan film with nano-silicon dioxide. Journal of Food Engineering, 113(3), 408-414. DOI: https://doi.org/10.1016/j.jfoodeng.2012.06.021

Zhang, D., Wang, H., Hu, Y. \& Liu, Y. (2015a). Chitosan controls postharvest decay on cherry tomato fruit possibly via the mitogen-activated protein kinase signaling pathway.Journal of Agricultural and Food Chemistry, 63, 7399-7404. DOI: https://doi.org/10.1021/acs.jafc.5b01566

Zhang, L., Chen, F., Lai, L., Wang, H. \& Yang, H. (2018). Impact of soybean protein isolate-chitosan edible coating on the softening of apricot fruit during storage. $L W T$ - Food Science and Technology, 96(1), 604-611. DOI: https://doi. org/10.1016/j.lwt.2018.06.011

Zhang, Y., Zhang, M. \& Yang, H. (2015b). Postharvest chitosang-salicylic acid application alleviates chilling injury and preserves cucumber fruit quality during cold storage. Food Chemistry, 174(1), 558-563. DOI: https://doi.org/10.1016/j. foodchem.2014.11.106 